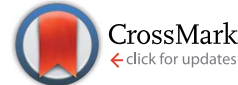

Cite this: Nat. Prod. Rep., 2015, 32, 723

\title{
Lessons from the Synthetic Chemist Nature
}

\author{
Gerrit Jürjens, Andreas Kirschning* and David A. Candito
}

Covering: 1981 to 2015

This conceptual review examines the ideal multistep synthesis from the perspective of nature. We suggest that besides step- and redox economies, one other key to efficiency is steady state processing with intermediates that are immediately transformed to the next intermediate when formed. We discuss four of nature's strategies (multicatalysis, domino reactions, iteration and compartmentation) that commonly proceed via short-lived intermediates and show that these strategies are also part of the chemist's portfolio. We particularly focus on compartmentation which in nature is found microscopically within cells (organelles) and between cells and on a molecular level on multiprotein scaffolds (e.g. in polyketide synthases) and demonstrate how compartmentation is manifested in modern multistep flow synthesis.

www.rsc.org/npr

syntheses can be measured. ${ }^{5}$ However, some of these concepts are restricted to individual chemical transformations and the underlying mechanisms to achieve selectivity. The Baran group has discussed and provided criteria that allow for comparison and even quantification of multistep synthesis. They cited three specific criteria: (a) overall yield, (b) step count and (c) percent ideality. ${ }^{5}$ The authors also acknowledge that the \% ideality should be used with caution and is more of a simple tool than a true overarching measure of efficiency. Today, these straightforward concepts are generally accepted within the synthetic community as they reflect an important step toward striving for improved efficiency.

In several aspects, nature can be seen as an ideal synthetic chemist. Understanding the biosynthesis of a natural product is often a productive starting point for the design of highly efficient, biomimetic total syntheses. ${ }^{6}$ Still, chemists have yet to achieve the same exquisite levels of selectivity, particularly, chemoselectivity, in the design of multistep processes. However, analysis of biosynthetic pathways reveals that atom-, redox- and step economies are not always well fulfilled in nature although from the synthetic chemist's point of view biosyntheses of complex natural products are regarded to be highly efficient. This may sound like a contradiction.

Herein, we discuss four fundamental strategies that are shared in both synthetic chemistry and nature, in so doing, we wish to shed light on this seeming contradiction. Based on these strategies, it is interesting to see the interplay between the chemist and nature, where the chemist can be seen to borrow from nature and in certain cases even improve on nature's design. The hope of this highlight is to discuss 
these fundamental strategies in a broad context taking examples from biosynthesis and the state of the art in organic synthesis and presenting them side by side. To clarify the scope of this review is not about biomimetic synthesis and we have deliberately not chosen biomimetic chemical syntheses as this has been extensively reviewed elsewhere. ${ }^{6}$

In this unique setting we hope to inspire the synthetic chemist to think more on natural systems for inspiration. Too often does the chemist draw a line between themselves and biologists, we hope to encourage a blurring of the lines. $^{7}$

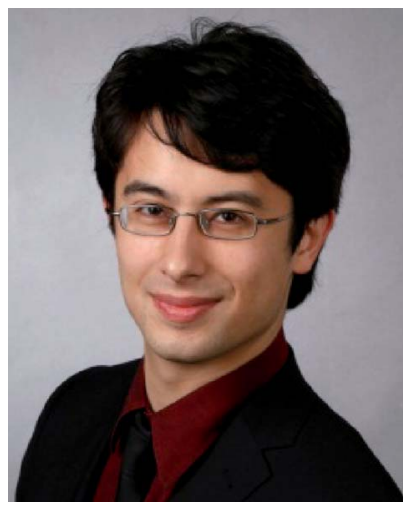

Gerrit Jürjens studied Life Science and Chemistry at the Leibniz University of Hannover and the Institute of Chemical \& Engineering Sciences, $A^{*}$ STAR (Singapore). He joined the group of Prof. Andreas Kirschning and received his $P h D$ in 2014, working in the field of total synthesis and biosynthesis. He is currently working on the lead structure optimisation of new antibiotic natural products in a Sanofi-Fraunhofer joint venture in Frankfurt as a post-doctoral researcher.

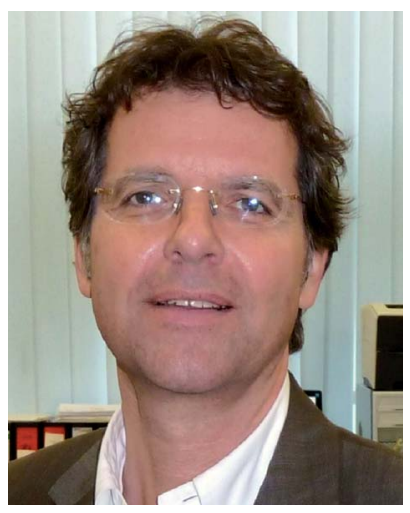

Andreas Kirschning studied chemistry at the University of Hamburg and at Southampton University (UK). In Hamburg, he joined the group of Prof. Ernst Schaumann and received his PhD in 1989 working in the field of organosilicon chemistry. After a postdoctoral stay at the University of Washington (Seattle, USA) with Prof. Heinz G. Floss, he started his independent research at the Clausthal University of Technology in 1991, where he finished his habilitation in 1996. In 2000 he moved to the Leibniz University Hannover. His research interests cover structure elucidation as well as the semi-, total and mutasynthesis of natural products, biomedical biopolymers, and synthetic technologies (solid-phase assisted synthesis, microreactors and inductive heating).

\section{Step- and redox-economies in nature}

In order to discuss the issue of step-economy in nature let us first take a look at erythronolide A (1a). It is composed of a macrolactone ring that is biosynthesised by a polyketide synthase type 1 (PKS). Starting from propionyl-CoA 14 transformations (additional four steps have to be added, if one starts counting from acetyl-CoA, additional six steps if transacylation steps from AT to ACP on the PKS are included, and additional seven steps if the activation and loading of the starter and elongation units are included) occur in a linear fashion to yield 6-deoxyerythronolide B (1b). After this, five additional biosynthetic tailoring steps fashion erythromycin A (2). ${ }^{8}$

So far, the first and only total synthesis of 2 was reported by Woodward and coworkers in 1981. This formidable synthesis was achieved in 69 (batch) steps (61 in the longest linear sequence) from commercially available starting materials. ${ }^{9}$ Several formal total syntheses of erythromycin precursors as well as of derivatives have been published since then. The most recent synthesis by Krische et al. elegantly furnished 6-deoxyerythronolide B (1) in only 14 linear steps (20 overall steps) in the longest linear sequence, clearly in fewer steps than nature does it. $^{10}$

However, the bioproduction of erythromycin A (2) is efficient because after about 60 hours Saccharopolyspora erythraea can provide the antibiotic in significant quantities. Examining the cyclic fed batch fermentation studies of Bushell and Lynch, ${ }^{\mathbf{1 1}}$ a

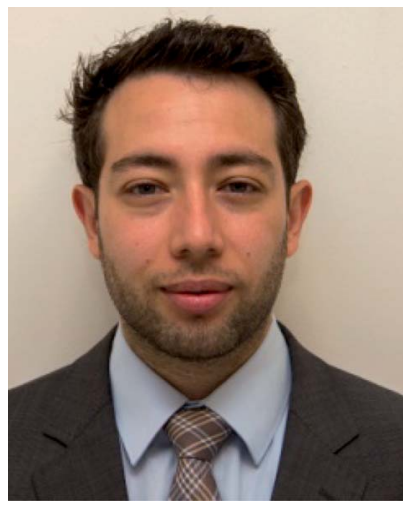

David completed his BSc Hon. at York University in 2007. During his honours work he was introduced to organic chemistry in the labs of Prof. Dr Michael Organ, where he worked on exploring the scope of PEPPSIIPr catalyzed Suzuki crosscouplings of aryl and alkyl halides with organoboranes. He was granted a PhD in organic chemistry from the University of Toronto in 2012. During his PhD he worked in the group of Prof. Dr Mark Lautens where his research spand the development of Catellani-type reactions for the synthesis of heterocycles and stereoselective reactions of arynes. The methodology he developed was applied to the synthesis of alkaloid natural products. David then went on to carry out postdoctoral research at the Leibniz University of Hannover under the supervision of Prof. Dr Andreas Kirschning from 2012 to 2014. During this time he worked on the biomimetic total synthesis of a polyketide known as elansolid $A$ and also worked on the development of ansamitocin-nanoparticle conjugates. While working in Germany, David was awarded an Alexander von Humboldt postdoctoral fellowship. David is currently employed as a Medicinal Chemist at Merck \& Co., Inc., where he is working in the Neuroscience area. 


\section{Biosynthesis}

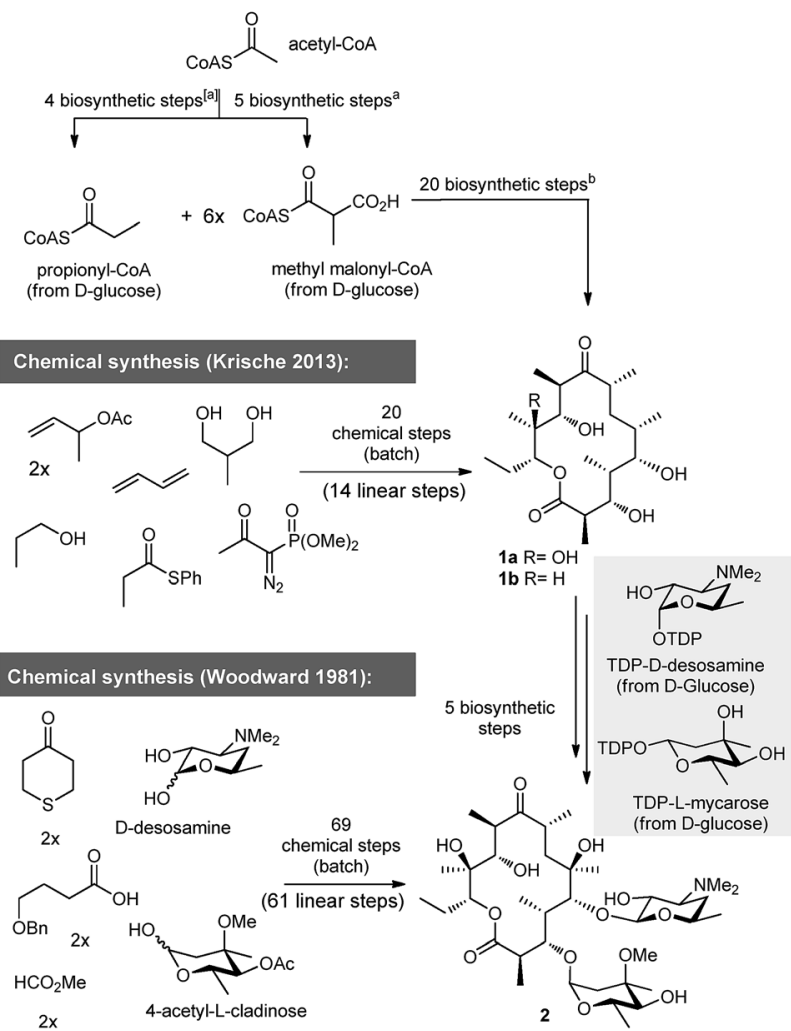

Scheme 1 Comparison of 6-deoxyerythronolide B (1) and erythromycin A (2) biosyntheses with Woodward's total synthesis of 2 and Krische's total synthesis of 1 ; ${ }^{a}$ step counts may vary because different biosynthetic routes are known; ${ }^{\mathrm{b}} 20$ steps when transacylation from AT to ACP is included.

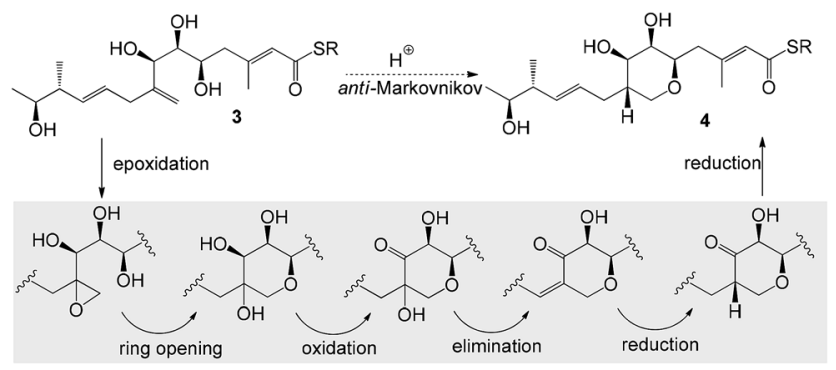

Scheme 2 Biosynthesis of the pyran ring in pseudomonic acids.

rough estimate on the productivity in $S$. erythraea is possible. About $0.18-0.53 \%$ of the glucose fed ends up in erythromycin (2). ${ }^{12}$ While this yield may not seem very impressive it is still significantly larger than the yield in the only total synthesis (less than $0.02 \%$ ). One gram of (dry) biomass produces up to $0.75 \mathrm{mg} \mathrm{h}^{-1}$ of 2 starting from $0.21 \mathrm{~g} \mathrm{~h}^{-1}$ glucose.

The biosynthesis ${ }^{13}$ of the pseudomonic acids, secondary metabolites originally isolated from Pseudomonas fluorescens NCIMB 10586, is an illustrative showcase for poor step- and redox-economy in nature (Scheme 2). A tetrahydropyran ring is a central structural element of the pseudomonic acids and starting from the advanced biosynthetic intermediate $\mathbf{3}$ it is formed by a six step sequence that includes two oxidations, two reductions an epoxide ring-opening and a dehydration to yield pyran 4. A chemist would likely suggest a single step alcohol addition onto the olefinic double bond in an anti-Markovnikov fashion. Despite these facts pseudomonic acids are produced by fermentation and are used under the name mupirocin against staphylococci and non-enteric streptococci.

One may speculate why many biosyntheses are linear and rather long, occasionally with poor redox-economy. From a chemists perspective the repertoire of reactions and building blocks nature can rely on is rather small. Additionally, it has been hesitant to develop new biosynthetic pathways. nature rather sticks to established multistep processes. Often iteration and the addition of "decorating" or "tailoring" steps lead to ever longer linear biosyntheses. From an evolutionary point of view long biosyntheses originate from genes that were incorporated into biosynthetic gene clusters and became finally operative, when the new metabolite provided improved survival for the producing organism. ${ }^{14}$

Unlike most chemical syntheses, biosyntheses are not batch but steady state processes in which intermediates are directly processed as they are formed. We believe that this fact is important to approach the topic of a perfect multistep synthesis from nature's perspective. Therefore, we shall begin with a "Gedanken-experiment" of a conventional multistep batch synthesis in order to be able to mirror it with nature's multistep processes before we discuss the four synthetic strategies.

\section{The flaw of multistep batch synthesis is its batch character}

Synthetic chemists commonly conduct multistep processes under batch conditions in which intermediates are formed in equimolar amounts and if necessary need to be isolated and/or further purified. Practically, batch mode synthesis is time consuming and uneconomical and here atom, step and redoxeconomies do matter.

In this context, the patented synthesis of olanzapine (7) is illustrative. ${ }^{15}$ Olanzapine is commercialised under the name ZYPREXA ${ }^{\circledR}$ and was among the top 20 best selling drugs

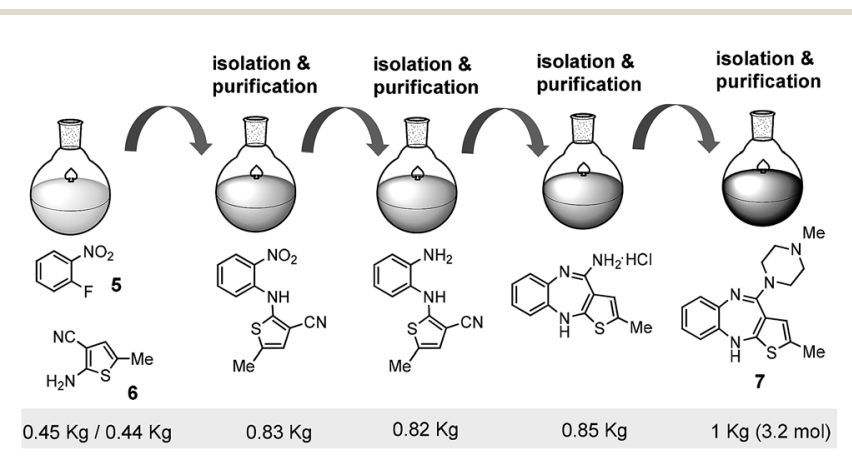

Fig. 1 The concept of multistep batch synthesis exemplified in a "Gedanken-experiment" (amount of materials in case of quantitative transformations). ${ }^{15}$ 


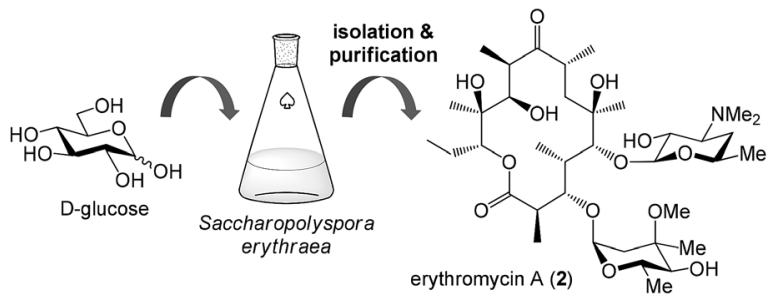

Fig. 2 A simplistic view on the bioproduction of erythromycin A (2).

worldwide in 2011. ZYPREXA® is an atypical neurolepticum and is used for the treatment of bipolar disorders and schizophrenia. ${ }^{16}$ It is known to exert antagonistic activity towards the dopamine receptor type 4 ( $\mathrm{D}_{4}$-receptor) and the serotonin receptor type 2 (5$\mathrm{HT}_{2}$-receptor). ${ }^{17}$ The patent ${ }^{15}$ describes a four step batch protocol starting from 1-fluoro-2-nitrobenzene (5) and 2-amino-5-methylthiophene-3-carbonitrile (6). Assuming the preparation of $1 \mathrm{~kg}$ (3.2 mol) of 7 with quantitative yield for each step (Fig. 1). This would require about $0.5 \mathrm{~kg}$ of starting materials 5 and 6 , and more than $0.8 \mathrm{~kg}$ of three intermediates are formed that need to be isolated. As a consequence multistep reaction sequences should ideally avoid work-up and isolation of intermediates.

At first glance the bioproduction of complex natural products such as erythromycin A (2) may look like a true batch protocol (Fig. 2). D-Glucose is the starting material, Saccharopolyspora erythrea acts as the "catalytic" system ${ }^{\mathbf{1 8}}$ and the fermenter resembles the flask or the reactor. Harvesting the bioreactor, yields the antibiotic 2 , still being used in the clinic.

The gross simplification depicted in Fig. 2 treats Saccharopolyspora erythrea as a black box or a batch reactor. Using the tools of molecular biology and biochemistry we can pry into these multicatalytic biosystems. In fact, the biosynthesis of erythromycin A (2) is a highly orchestrated multistep and rather linear steady state process, catalyzed by enzymes, many being part of a catalytic megacomplex, the polyketide synthase (PKS, vide infra). In contrast to the multistep batch process depicted in Fig. 1, these catalytic architectures circumvent the stoichiometric formation of intermediates.

Primary metabolism "feeds" the biosynthetic pathways of secondary metabolite production and is organised in a complex network of enzymatic transformations and routes. A biosynthetic intermediate is often generated from different sources and the intermediate can be the starting point for different

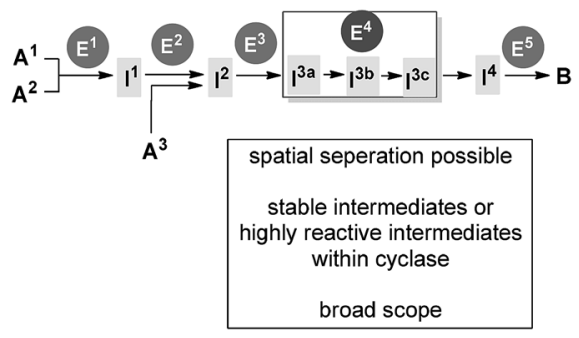

Fig. 3 Enzyme-catalyzed multistep biosynthesis $\left(A^{1}-A^{3}=\right.$ substrates, building blocks, $B=$ product of biosynthesis/natural product; $1^{1}-I^{4}=$ reactive intermediates, $E^{1}-E^{5}=$ enzymes, $E^{4}=$ enzyme that catalyzes a multistep process via highly reactive intermediates $\left.\mathrm{I}^{3 \mathrm{a}}-\mathrm{I}^{3 \mathrm{c}}\right)$. biosynthetic routes as exemplified in Fig. 3. In most cases, building blocks A are activated carboxylic acids such as acetylor propionyl-CoA, aminoacyl phosphates or alkyl diphosphates.

Often tailoring transformations such as acylations, alkylations or glycosylations implement further "decoration" into the backbone (e.g. $\mathbf{I}^{\mathbf{4}} \rightarrow \mathbf{B}$, Fig. 3). Intermediates I are difficult to detect or to track, so that first insights into biosynthetic pathways came from the isolation of biosynthetic end product $\mathbf{B}$, while manifestation of intermediates I was necessary to provide a detailed description of the pathways. ${ }^{19}$ These biosyntheses either rely on a cocktail of individual enzymes (e.g. in terpene biosynthesis) ${ }^{20}$ or are based on megaenzyme complexes such as the polyketide synthases that are e.g. part of erythromycin (2) biosynthesis, or non-ribosomal peptide synthases (NRPS). In both cases the templating property of PKS and NRPS guarantee spatial separation or compartmentation of each step (see also chapter 4.4).

Now that we have provided an overview of secondary metabolite biosynthesis, with an emphasis on polyketides biosynthesis, we will go on to discuss four strategies that nature has developed in order to allow for efficient multistep synthesis with seeming disregard to the chemists notion of step count and atom economy. Typically, the strategies are based on steady state processes and are (a) multicatalysis, (b) domino/cascade reactions, (c) iteration and (d) compartmentation. We discuss compartmentation in greater detail ${ }^{\mathbf{2 1 , 2 2}}$ as this aspect can hardly be found in the literature yet. ${ }^{3}$ Overall, these four strategies add to those justified and often discussed arguments that are based on protecting group chemistry and the high chemo-, regio- and chemoselectivities of biocatalysts. ${ }^{23}$

\section{Nature's synthetic strategies are based on steady state processes}

\subsection{Strategy 1: continuous processing by multicatalysis}

As previously discussed, nature relies on linear steady state processes, in which individual enzymes are responsible for single transformations. The early steps of terpene biosynthesis

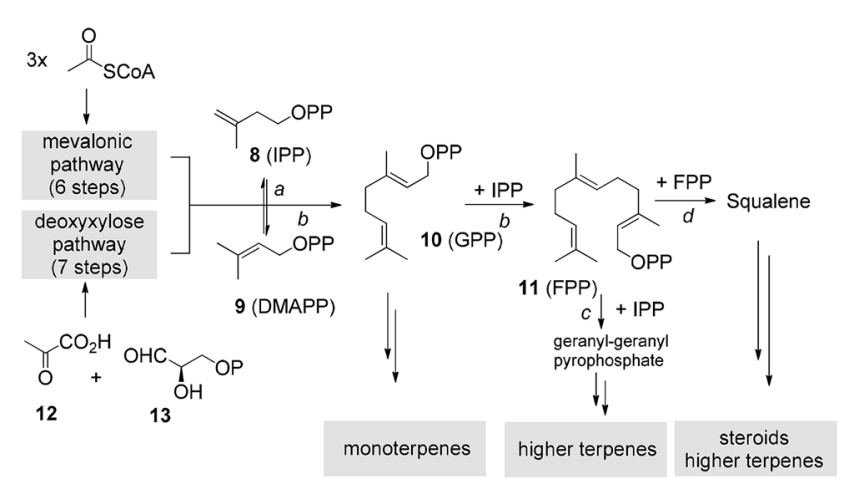

Scheme 3 Overview on the biosynthesis of acyclic terpene pyrophosphates, a typical example of a biological multicatalytic system [IPP = isopentenyl pyrophosphate (8); DMAPP = dimethylallyl pyrophosphate (9); GPP = geranyl pyrophosphate (10); FPP = farnesyl pyrophosphate (11) (enzymes involved: (a) isopentyl-diphosphate deltaisomerase; (b) farnesyl diphosphate synthase; (c) geranylgeranyl diphosphate synthase; (d) farnesyl-diphosphate farnesyltransferase)]. 


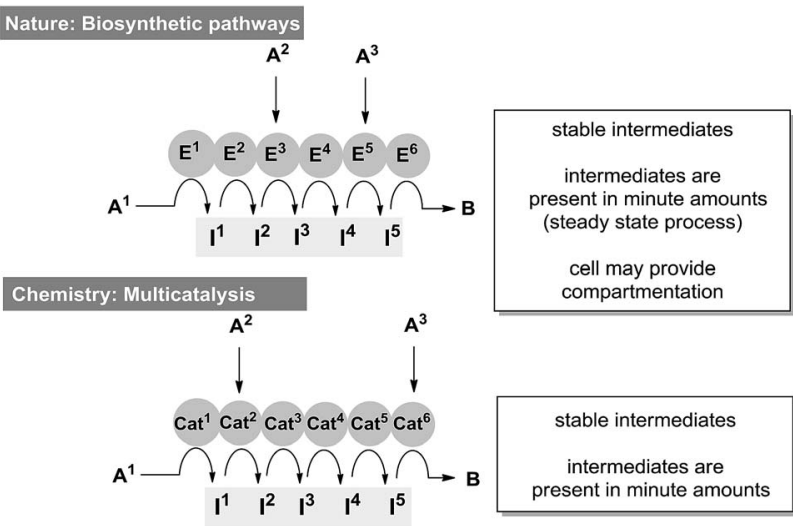

Fig. 4 Multicatalytic systems in biosynthesis and chemical synthesis.

are a typical example for a multicatalytic biosynthetic pathway (Scheme 3). Two distinct biosynthetic pathways, namely the mevalonate and the deoxyxylulose route, ${ }^{24}$ provide nature's isoprene analogues isopentenyl pyrophosphate [IPP (8)] and dimethylallyl pyrophosphate [DMAPP (9)]. The former pathway utilises six enzymes to reach IPP starting from SCoA activated acetic acid, while the latter route requires seven enzymes starting from pyruvate (12) and glycerinaldehyde-3-phosphate (13). Then, an iterative process provides geranyl pyrophosphate [GPP (10)], farnesyl pyrophosphate [FPP (11)], geranyl geranyl pyrophosphate and squalene. Each of these advanced intermediate building blocks represents one of the terpene classes (e.g. mono-, di-, sesqui-, tri- and tetraterpenes).

Multicatalytic reactions and sequences have also emerged in organic synthesis. These systems could be purely based on biocatalysts and synthetic catalysts or on biocatalyst/synthetic catalyst combinations. ${ }^{25}$ Within the category of synthetic catalysts further subdivisions can be made based upon whether the catalyst is an organocatalyst or based on inorganic/organometallic catalysts (Fig. 4).

The combination of multiple catalysts has several benefits such as reduced waste, reduced overall time to realise multistep syntheses, the ability to control unstable intermediates and the ability to generate molecular complexity from simple precursors. However, certain points must be taken into account, such as the compatibility of starting materials/intermediates and catalysts with each other and with the reaction conditions (temperature, solvent, pressure etc.). Additionally, the relative rates of the individual processes, and the selectivity of the individual catalysts must be considered, such that reactions occur in a definite sequence on precisely the intermediate which one intends. Despite many parameters needing to be controlled", researchers have begun to uncover reactions and strategies which already led to several impressive examples.

An exciting new field in the arena of multicatalysis is the use of several metal catalysts that operate orthogonally in one pot and provide highly complex molecular architectures from simple precursors. Jeong and co-workers ${ }^{26}$ reported one of the first examples of a multicatalytic palladium/rhodium system with multiple $\mathrm{C}-\mathrm{C}$ bonds being formed (Scheme 4). Initially, a

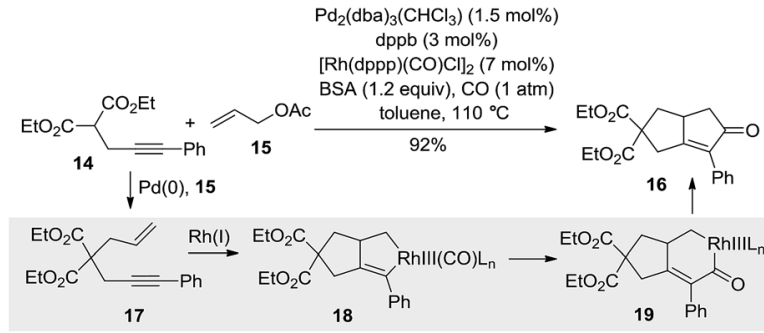

Scheme 4 Multicatalytic Pd-Rh-promoted allylic alkylation-PKR (for purposes of clarity, only key intermediates are depicted).

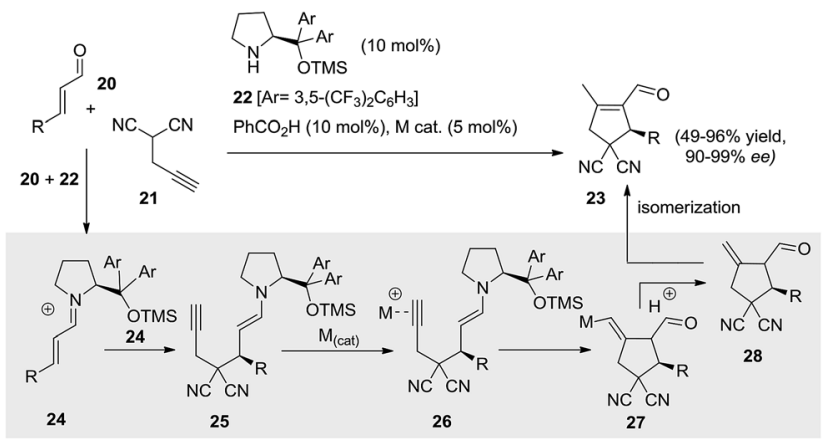

Scheme 5 Dual metal-organocatalytic synthesis of cyclopentene carbaldehydes 23; Mcat. $=\mathrm{Cu}(\mathrm{OTf})_{2}(5 \mathrm{~mol} \%) / \mathrm{PPh}_{3}(20 \mathrm{~mol} \%)$, or CuOTf (5 mol\%)/PPh 3 (20 mol\%), or Au(NTf $)$ ( 5 mol\%) (for purposes of clarity, only key intermediates are depicted).

palladium-catalyzed allylation between malonyl derivative $\mathbf{1 4}$ and allyl acetate 15 takes place to furnish intermediate 17. This intermediate enters a second catalytic cycle where rhodium triggers a Pauson-Khand type reaction (PKR). Via intermediates 18 and 19 the bicyclic ring system 16 is generated in excellent yield. The authors found that the chosen rhodium precatalyst is critical to the success of the multicatalytic cascade. This example demonstrates the multitude of opportunities chemists have compared to nature when developing multistep sequences, as there are no analogous catalytic reactions known in nature.

Several examples of cooperative utilisation of transition metal catalysts, organocatalysts and even enzymes in one-pot have appeared in the literature. Jørgensen $e t$ al. showed that proline-derivative 22 and a $\pi$-Lewis acid based on gold or copper, can affect the reaction of propargyl malononitrile 21 with $\alpha, \beta$-unsaturated aldehydes 20 , to furnish highly enantioenriched cyclopentene carbaldehydes 23 (Scheme 5). ${ }^{27}$

Initially, the organocatalyst is responsible for iminium formation and directs highly selective 1,4-addition (intermediates $\mathbf{2 4}$ and 25). Then, the $\pi$-Lewis acid takes over (intermediate 26) disposing the alkyne toward intramolecular 5-exo-dig cyclisation to provide chiral cyclopentene carbaldehydes (intermediates 27 and 28). From control experiments it became evident that during transformation of $\mathbf{2 6}$ to $\mathbf{2 7}$ the two catalysts operate cooperatively.

As early as 2002 a multicatalytic system was reported that utilises an enzyme, an organocatalyst and a metal catalyst in 


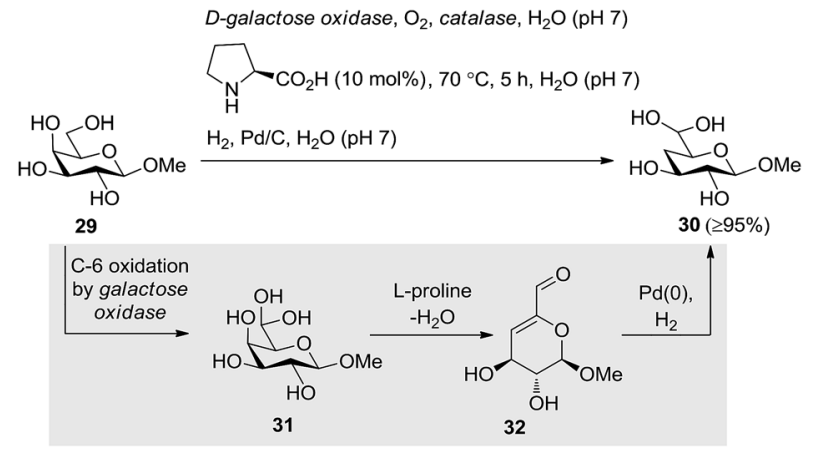

Scheme 6 One-pot triple catalysis for the conversion of methyl $\beta$-Dgalactoside (29) to methyl 4-deoxy-6-aldehydo- $\beta$-D-galactoside (30).

water as the solvent in one-pot (Scheme 6). ${ }^{28}$ The conversion of methyl $\beta$-D-galactoside (29) to methyl 4-deoxy-6-aldehydo- $\beta$-Dgalactoside (30) was initiated by the action of D-galactose oxidase that affects the oxidation at C-6. The resulting aldehyde (intermediate 31), in equilibrium with its hydrate, was warmed up and L-proline promoted dehydration to form the corresponding enal (intermediate 32). Finally, hydrogenation over palladium on charcoal gave the deoxygenated hexoside $\mathbf{3 0}$ in excellent yield. This process proceeded without purification or isolation of intermediates, only reagents are added and conditions are altered. Remarkably, this process does not require protection of hydroxyl groups and it demonstrates the power of multicatalysis in multistep processes.

\subsection{Strategy 2: domino reactions}

Domino or cascade reactions have emerged as an important tool for the organic chemist and over the past 20 years there has been a great deal of research in this area and a wealth of new processes have become available. As defined by Tietze, a domino reaction is a process whereby a starting material passes through two or more intermediates on its way to the final product, in a sequential manner, where functionality generated in the first step allows for further transformations to take place. ${ }^{29}$ The whole process occurs under the same reaction conditions and commonly all components are present from the very beginning (Fig. 5 ).

Nature also relies on this concept and in this context terpene cyclases are excellent examples. ${ }^{30}$ These enzymes have the ability to control the reactivity of intermediate carbocations of a domino sequence that results in the large number of known oligocyclic terpenes. During cyclisation the ring sizes and the configuration of stereogenic centers formed are dictated by the defined folding of the linear pyrophosphate precursor and governed by stereoelectronic effects in the enzyme active site, where "special" conformations can be stabilised. Commonly, the domino sequence is initiated by formation of a 3,3-disubstituted allyl cation which reacts intramolecularly with one of the trisubstituted alkene moieties of the substrate. A new carbocation is formed which commonly has three options: (a) internal or external trapping of a nucleophile, (b) deprotonation

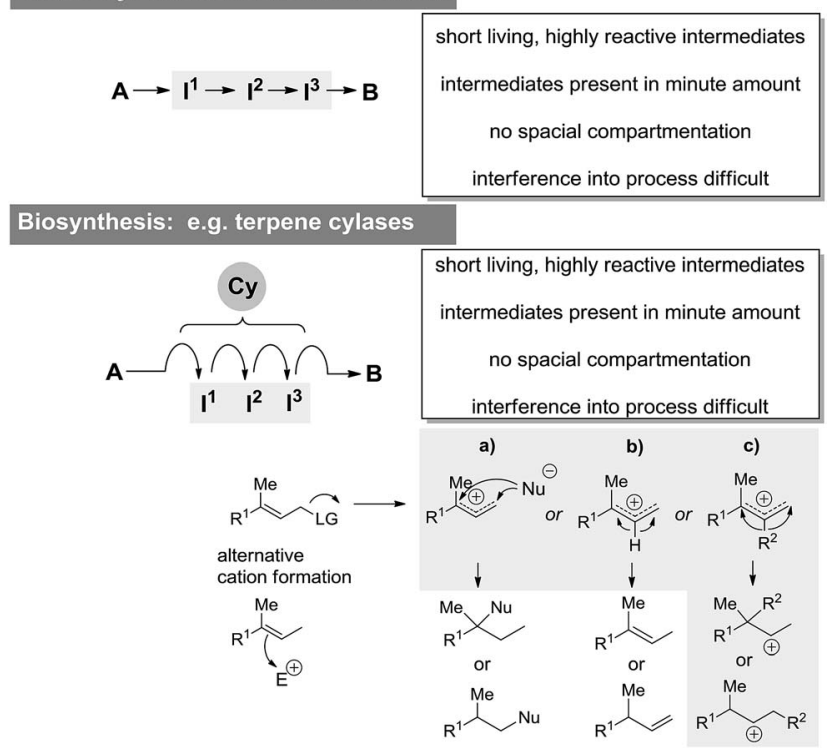

Fig. 5 Principal concept of domino reactions and comparison to terpene cyclases; $(\mathrm{A}=$ substrate of terpene cyclases, $\mathrm{B}=$ cyclisation product; $I^{1}-I^{3}=$ reactive intermediates, $\mathrm{Cy}=$ terpene cyclase).

and formation of an alkene or (c) Wagner-Meerwein rearrangement and formation of a new carbocation (Fig. 5). ${ }^{31}$

The taxadiene synthase that operates in the biosynthesis of the yew tree (Taxus brevifolia) metabolite paclitaxel (35, TAXOL $\left({ }^{\circledR}\right)$ is an illustrative example. Paclitaxel inhibits microtubule depolymerisation ${ }^{32}$ and commercially is one of the most successful anti-cancer compounds. This diterperne is based on a 6-8-6 tricyclic carbon backbone which is formed from geranylgeranyl pyrophosphate (33) in an enzyme-catalyzed domino
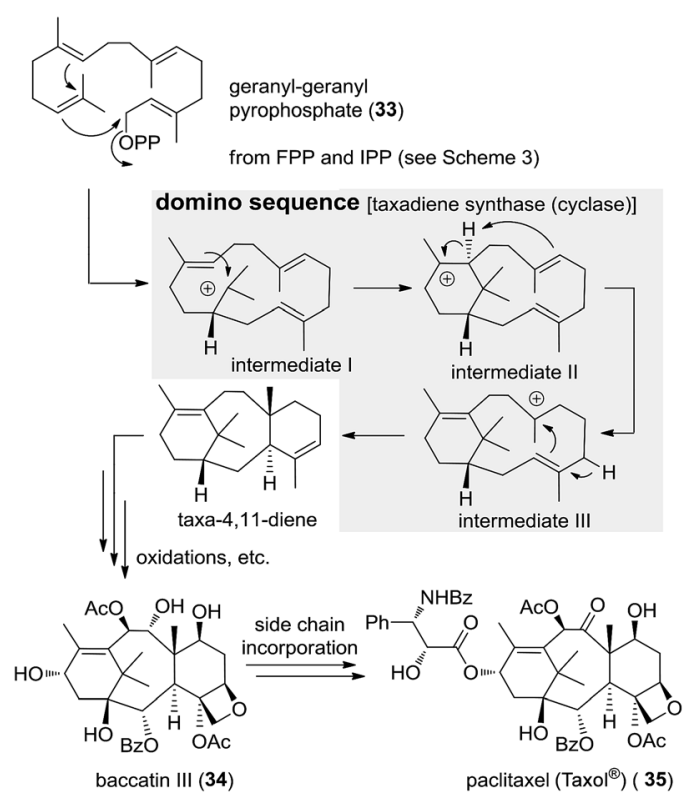

Scheme 7 Biosynthesis of paclitaxel (38) (cationic intermediates are positioned on grey background). 


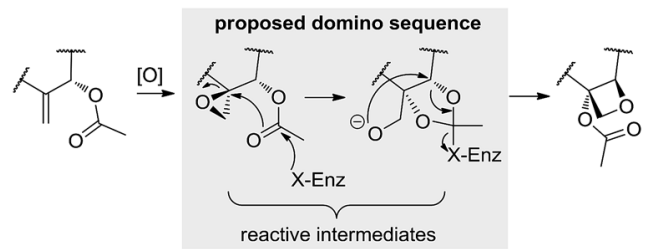

Scheme 8 Proposed domino sequence in the formation of the oxetane ring in paclitaxel (35).

reaction (Scheme 7). The cascade proceeds through several cationic intermediates I-III formed either by $\pi$-solvolysis, elimination or $\mathrm{H}$-shifts. A series of tailoring oxidations and esterifications provide paclitaxel via baccatin III (34). Within this biosynthesis, another remarkable domino sequence occurs during the tailoring phase of the taxane backbone to form the oxetane ring as depicted in Scheme 8. $^{33}$

Cationic intermediates also drive iminium biosyntheses of alkaloids. Anionic species govern cascade sequences in polyether and ladder polyether biosyntheses as studied in detail for monensin. ${ }^{34}$ Radical cascade reactions are not very widespread in nature. Often these sequences are short and represent rearrangements commonly initiated by $S$-adenosyl methionin or by vitamin $\mathrm{B}_{12}$.

In a similar fashion chemists have utilised this concept of creating reactive intermediates based on carbocations, carbanions and $\mathrm{C}$-centered radicals to build up several carbocyclic systems in one step.

An impressive example was disclosed by the Kilburn group, ${ }^{35}$ in which $\mathrm{SmI}_{2}$ is used to reductively initiate a radical cascade of cyclisation-ring expansion-cyclisation via proposed intermediates 39-41 starting from cyclopropyl ketone 36. The use of HMPA is supposed to influence the respective size of the coordinating samarium, thus stabilizing either intermediate I or II and hence controlling the relative stereochemistry of the product $\mathbf{3 7}$ and $\mathbf{3 8}$, respectively (Scheme 9 ).

Although this domino reaction differs from terpene cyclasepromoted biosyntheses in that radicals instead of carbocations

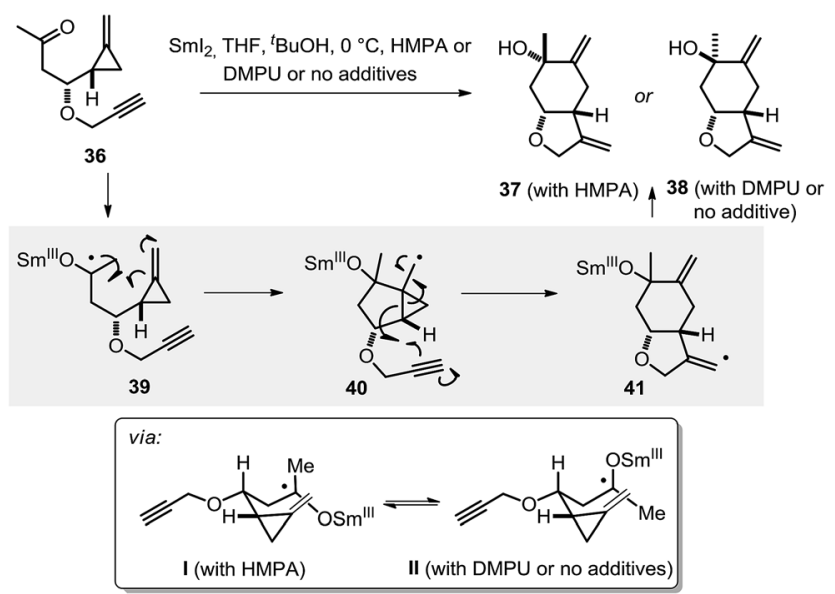

Scheme $9 \mathrm{Sml}_{2}$ promoted cyclisation-ring expansion-cyclisation cascade by Kilburn et al. ${ }^{35}$

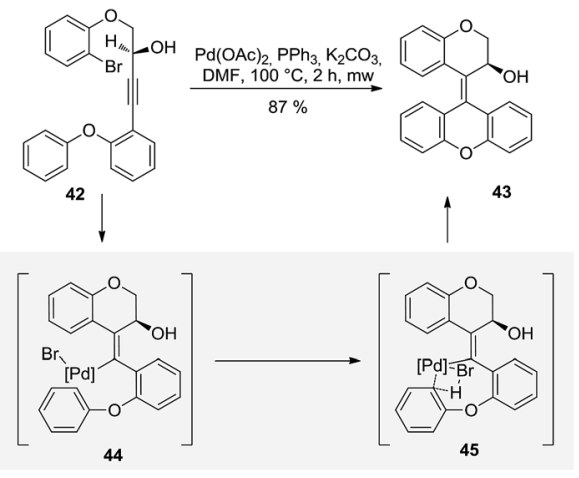

Scheme 10 A combined $\mathrm{Heck} / \mathrm{C}-\mathrm{H}$ activation. ${ }^{36}$

are the highly reactive intermediates, the underlying principles of domino reactions are alike. In the case of terpene cyclases the resulting stereochemistry is controlled by the asymmetric environment provided by the biocatalyst which exerts a defined conformation of the substrate. In the Kilburn example the starting material prefolds to a preferred conformer in solution, which can further be adjusted by choice of additional ligands on the samarium species.

This concept of creating a reactive intermediate which initiates a second very different reaction is rather common in transition metal catalysis. Recently, Tietze et al. published an example of a palladium catalyzed carbopalladation-C-H activation sequence (Scheme 10). ${ }^{36}$ The reaction starts with $\mathrm{Pd}$ insertion into the carbon halogen bond followed by a 6-exo-dig cyclisation through carbometalation of the triple bond to furnish intermediate $\mathbf{4 4}$. The close proximity of the aryl group to the metal center initiates a $\mathrm{C}-\mathrm{H}$ insertion of the palladium, resulting in the formation of the product $\mathbf{4 3}$ after reductive elimination.

\subsection{Strategy 3: iterative reactions}

Iteration of steps as was discussed for the acyclic terpene pyrophosphates in Scheme 3, is a third concept how nature simplifies biosynthetic processes.

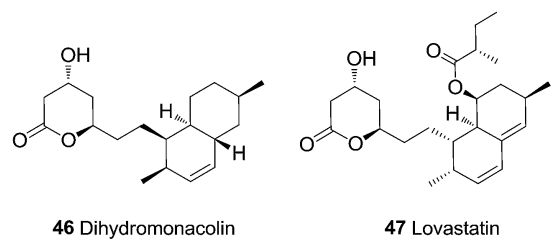

Indeed, modularity is widespread in nature and can also be found in polypeptides, oligonucleotides and oligosaccharides, indeed most natural small-molecules are biosynthesised via iterative coupling reactions. In contrast to bacterial type 1 polyketide synthases that are arranged in a complex modular fashion (see also erythromycin PKS in chapter 4.4), fungal polyketide synthases use only a simplified enzymatic machinery that is still able to create large and complex molecular architectures. The key for understanding these fungal PKS modules is their ability to operate iteratively. ${ }^{37}$ The exact mechanism of 


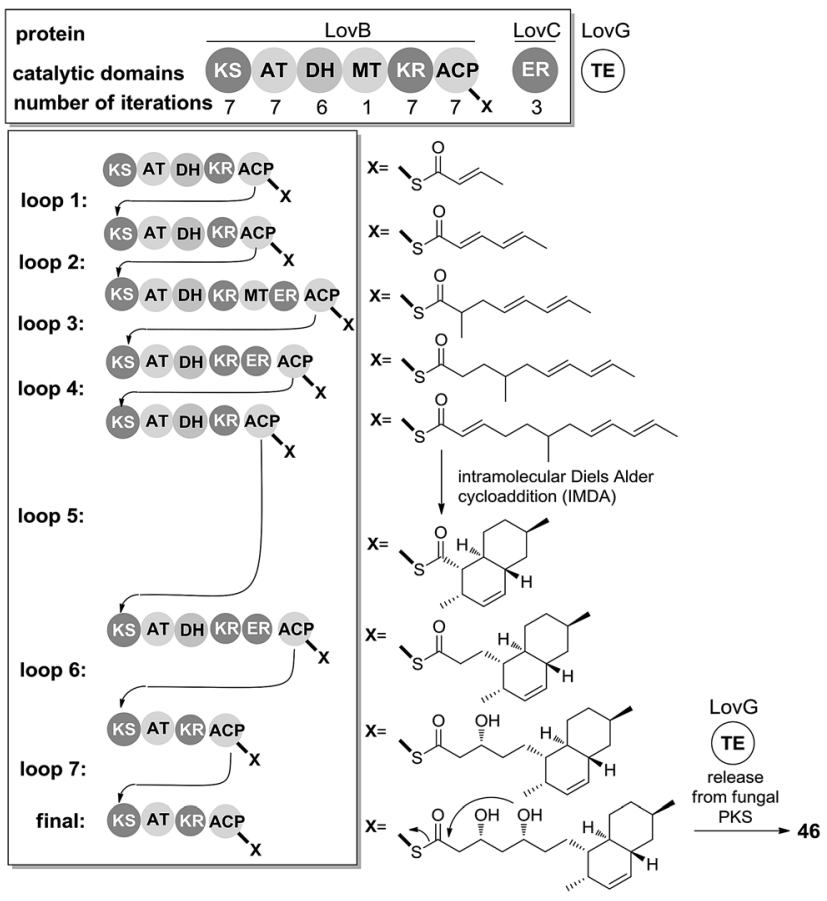

Scheme 11 Dihydromonacolin (46), a precursor of the hypolipidemic drug lovastatin 47 and the iterative biosynthesis of 46 (KS = ketosynthase, $\mathrm{AT}=$ acyl transferase, $\mathrm{DH}=$ dehydratase, $\mathrm{MT}=$ methyl transferase, $\mathrm{KR}=$ ketoreductase, $\mathrm{ACP}=$ acyl carrier protein, $\mathrm{ER}=$ enoyl reductase; $\mathrm{TE}=$ thioesterase $\mathrm{X}=\mathrm{ACP}$-bound intermediates).

chain extension is largely unknown but their impressive iterative power is demonstrated in the reconstitution of the PKS of dihydromonacolin (46). Compound 46 is a precursor of the hypolipidemic drug lovastatin $\mathbf{4 7}$ that is produced by Aspergillus terreus (Scheme 11). ${ }^{38 a}$

The catalytic domains are organised in the one multifunctional (LovB) and one monofunctional protein (LovC) that are responsible for the biosynthesis of 46. The individual enzymatic units within the domains are iteratively used up to eight times before release from the PKS. ${ }^{38 b}$ The final product 46 is formed after a bioprocess consisting of seven iterations that only differ in the number of enzymatic units operating and the length of the carbon backbone of the ACP-bound intermediates. As a result only seven different enzymes are required to perform 21 reactions. At first glance, the iterative process is not revealed in the structure of 46. But the structural complexity is further increased because of an intervening intramolecular Diels-Alder cycloaddition, which most likely proceeds in a spontaneous manner (Scheme 11).

Polyketides of type II are formed by even more simplified PKS variants which are composed of only $\mathrm{KS}_{\alpha}, \mathrm{KS}_{\beta}$ and ACP domains. ${ }^{39}$ The $\mathrm{KS}_{\alpha}$ and the ACP domains work in an iterative sense, while $\mathrm{KS}_{\beta}$, also known as chain initiation factor, programming the final length of the polyketide chain as $\mathrm{KS}_{\beta}$ converts malonyl ACP to acetyl CoA to form the starter unit. Optionally, other domains can be present such as ketoreductases (KR), cyclases (CYC) and aromatases (ARO). Interestingly, these enzymes are not an element of the PKS but are

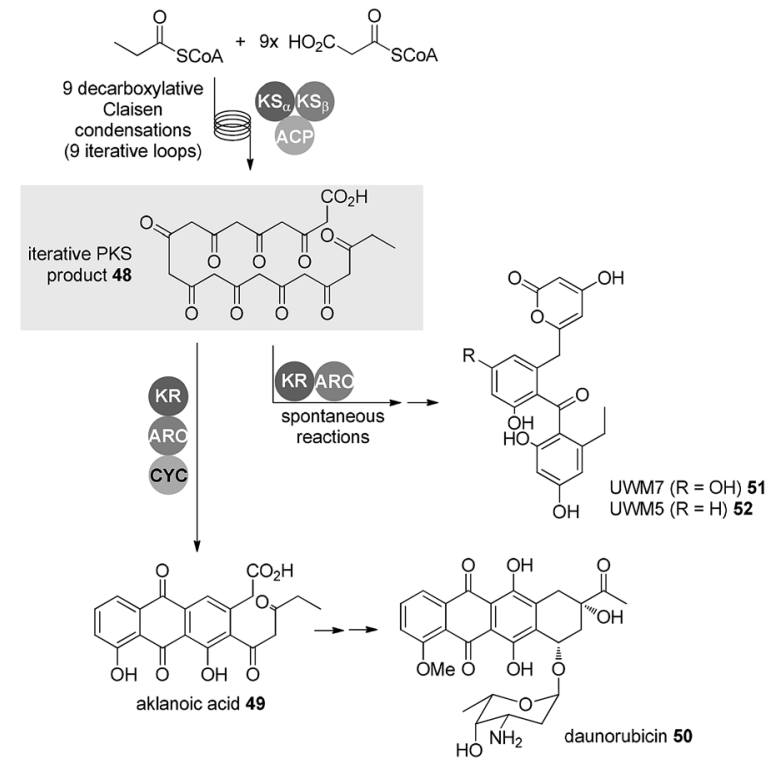

Scheme 12 Iterative biosynthesis of PKS type II decaketides to daunorubicin 50. Lack of the cyclase DpsY results in formation of UWM5 (52) and UWM7 (51).

associated with it and serve to modify the linear polyketide chain.

In Scheme 12 the iterative process for the biosynthesis of decaketides is summarised. Starting from one propionyl-CoA and nine malonyl-CoA extender units, nine iterative loops yield the intermediate polyketone which can be further processed to a variety of different carbon skeletons that are subsequently processed to anthracycline antibiotics such as daunorubicin (50). Although the factors and proteins governing and controlling the number of iterations are not fully understood yet, it is discussed that folding of the substrate in the enzyme pocket is crucial and once a certain chain length is reached the iterative process stops. Noteworthy, the biosynthesis nicely demonstrates the high reactivity of intermediate $\mathbf{4 8}$ - a lack of the cyclase results in spontaneous cyclisation to other products such as $\mathbf{5 1}$ and $\mathbf{5 2 . { } ^ { 3 9 , 4 0 }}$

Iterative sequences have also been developed by chemists. This strategy provides complexity in a modular fashion from simple precursors. In analogy to iteration by fungal PKS or type II PKS, the Yamamoto group found that supersilyl enol ethers 53

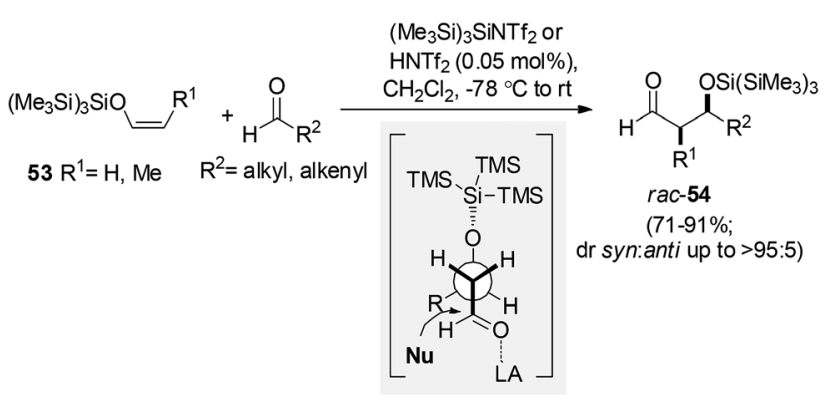

Scheme 13 Iterative aldol reactions and proposed transition state for syn-product. ${ }^{41}$ 


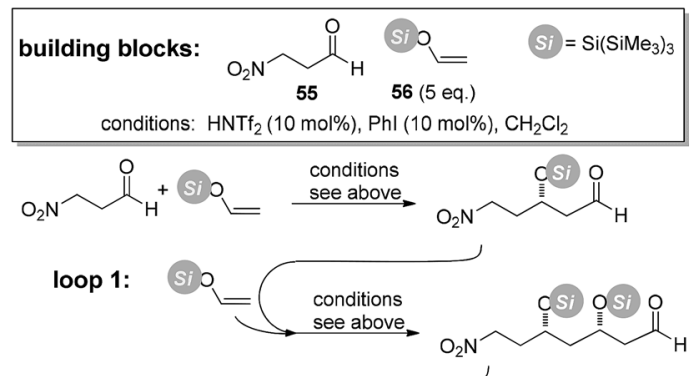

loop 2:
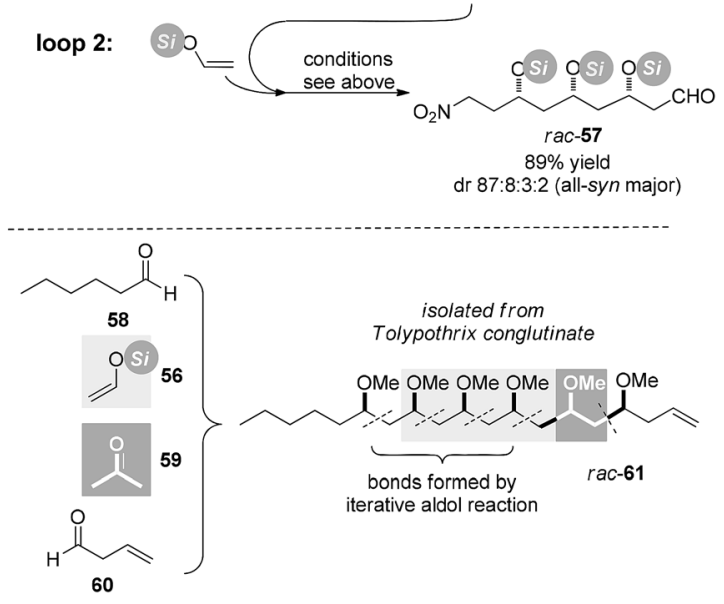

Scheme 14 Iterative triple aldol reaction and in the total synthesis of the polymethoxy-1-alkene rac-52. ${ }^{42}$

can be utilised for iterative aldol processes with aldehydes to yield aldol products 54 with high syn-selectivity (Scheme 13). ${ }^{\mathbf{4 1}}$

Although a new aldehyde is formed, the second aldol reaction is retarded due to the large size of the supersilyl group. Additives such as PhI facilitated oligoaldol processes. For example one triple aldol reaction provided polyketide-type triol 57 from silyl enol ether $56(\mathrm{R}=\mathrm{H})$ and 3-nitro propanal 55 (Scheme 14). It was postulated that organoiodides react with $\left(\mathrm{Me}_{3} \mathrm{Si}\right)_{3} \mathrm{SiNTf}_{2}$ to furnish a cationic, sterically less congested complex of the type RI-Si($\left(\mathrm{SiMe}_{3}\right)_{3}{ }^{+} \mathrm{NTf}_{2}{ }^{-}$. The iterative process was successfully applied ${ }^{\mathbf{4 3}}$ in the synthesis of the polymethoxy-1alkene $\mathbf{6 1}$ that was, among other metabolites, isolated from the polytoxin producing blue-green algae Tolypothrix conglutinate. Here, this aldol reaction proceeds three times before being terminated due to the steric congestion present in the product. 1-Iodo-2-phenylacetylene serves as a mediator. ${ }^{44}$ The fact that structural parameters such as bulkiness lead to termination of the iterative cycle also likely play a role in type 2 PKS biosynthesis as discussed above.

The group of Martin Burke developed a powerful methodology based upon iterative cross-coupling reactions of bifunctional MIDA-boronates (Scheme 15). In principal, the polarity of elements $n$ or $i$ can be flexibly chosen such that selective crosscoupling occurs in a stereoretentive sense to give the adduct $n+$ $i$, deprotection and activation of the boronate then takes place after which time additional steps may invert the polarity of the substrate. Possible options are the conversion of the vinyl boronate into a vinyl halide or the formation of pinacol boronates. This cycle can be repeated several times to access fragments in

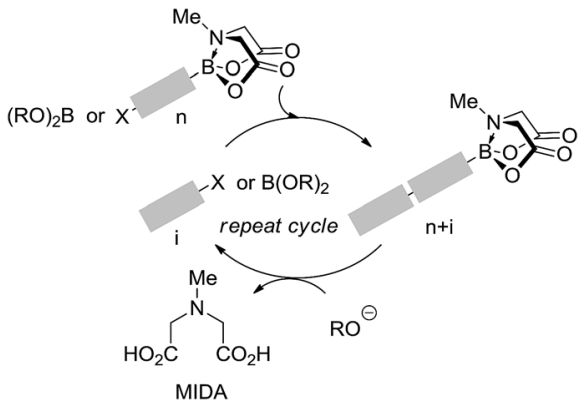

Scheme 15 Concept of iterative cross-coupling with MIDAboronates.

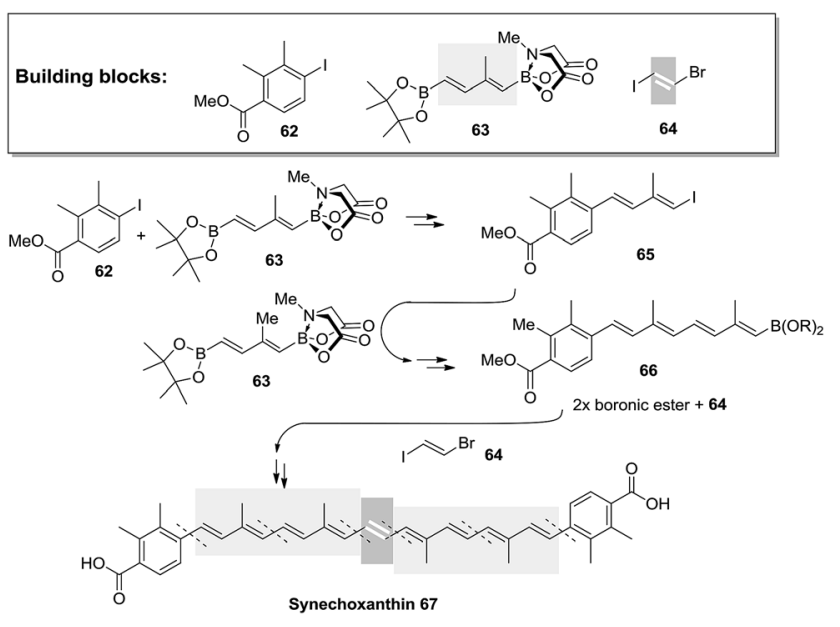

Scheme 16 Synthesis of synechoxanthin (67) using MIDA-boronate building blocks $62-64$.

stereochemically pure form bearing appropriate functionality for further reactions. The different principal options of this new methodology becomes evident when studying the total syntheses of the polyenes synechoxanthin (67) (Scheme 16). ${ }^{45}$

The synthesis of synechoxanthin (67) started from aryl iodide 62 by Suzuki-Miyaura cross coupling with mixed boronate 63 . The resulting vinyl MIDA boronate moiety was transformed into the corresponding iodide $\mathbf{6 5}$ so that the same elongation process with building block 63 could be repeated again. The resulting polyenyl MIDA-boronate was activated as boronic ester 66 for the dimerisation step with dihalo alkene 64. In a remarkable recent paper the Burke group demonstrated that 12 different MIDA building blocks are in principal sufficient to iteratively prepare about $75 \%$ of all known polyene natural products. $^{46}$

\subsection{Strategy 4: spatial compartmentation of individual steps - from polyketide biosynthesis to flow chemistry}

The fourth strategy of nature's multistep biosynthesis is spatial compartmentation which can be discussed from two perspectives, a microscopic ${ }^{47}$ and a molecular view. Microscopic compartmentation refers to cell organelles which have individual metabolic functions and which are characterised by 


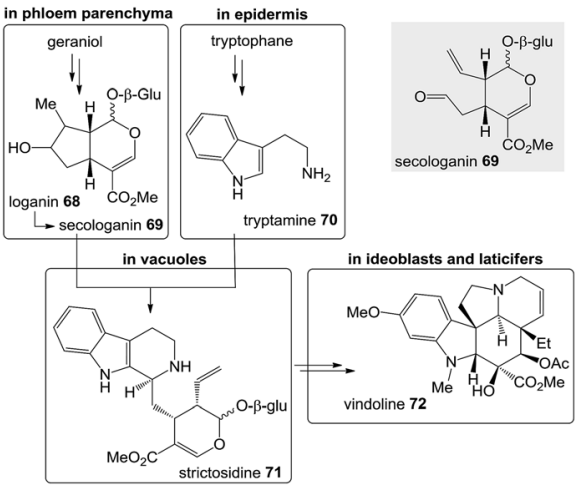

Scheme 17 Compartmentation in the biosynthesis of plant derived (Catharanthus roseus) indole alkaloid vindoline 72 .

different chemical conditions with respect to enzyme compositions and $\mathrm{pH}$. Building blocks and metabolites are transferred between cell organelles or even between different cell types as found in plants by active transport systems or passively by e.g. osmosis. In that context organelles are chemical reactors which form a network with active mass transport among them. Microscopic compartmentation has been studied in plants to some degree..$^{21}$ For example, in Catharanthus roseus both intraand intercellular compartmentation play an important role in the biosynthesis of different types of terpenoid indole alkaloid skeletons such as vindoline $\mathbf{7 2}$, which is a precursor of the commercially important anticancer agents vinblastine and vincristine (Scheme 17). ${ }^{48}$

The early terpenoid precursors such as loganin $\mathbf{6 8}$ from the mevalonate terpenoid pathway are made in internal phloem parenchyma, the other important precursor tryptamine $\mathbf{7 0}$ in the epidermis. The last step of the terpenoid precursor secologanin 69 occurs in epidermis cells. Strictosidine synthase is present in the vacuoles and catalyzes the condensation of tryptamine 70 and secologanin 69 to yield the intermediate strictosidine $\mathbf{7 1}$. Finally, vindoline $\mathbf{7 2}$ is formed in other specialised cells, the ideoblasts and laticifers so that an intercellular transport of strictosidine must take place and the biosynthesis is compartmented. In essence, the flux through a pathway is controlled by transport from the site of production of a precursor to the site of the next enzyme.

The idea of compartmentation and passive transport is also found in synthetic chemistry at the interphase of two phases which can actively be promoted by phase transfer catalysts. E.g., this concept has been exploited for increasing the life time of free radicals using micelles as reactor compartments. ${ }^{22}$

Spatial separation can also be realised on a nanoscopic scale, where template structures based on e.g. multiple protein complexes are able to separate biosynthetic intermediates from each other. Such protein complexes are specifically folded bringing various catalytic domains in close proximity thereby allowing bound biosynthetic intermediates to be processed by transfer to the next catalytic domain (Fig. 6).

As mentioned above, erythromycin A (2) is a classical example of a complex antibiotic produced by Saccharopolyspora

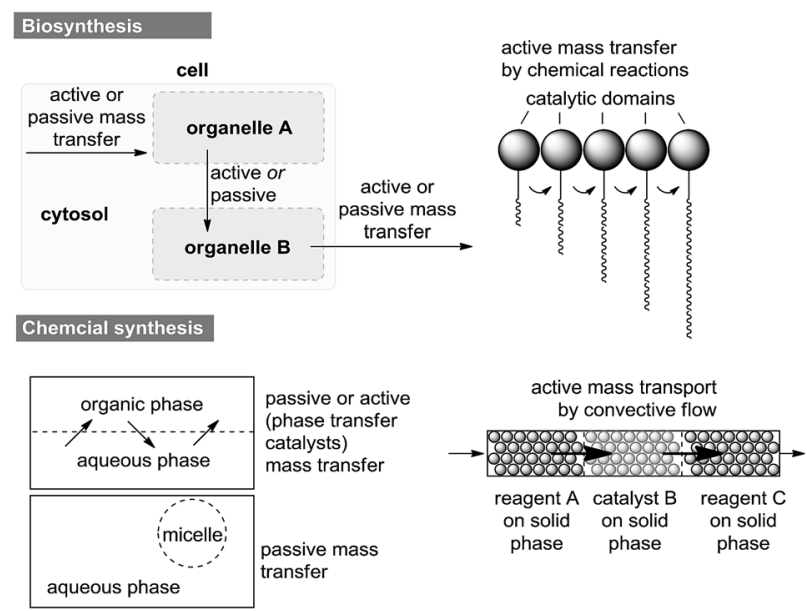

Fig. 6 Examples of compartmentation in nature and in chemical synthesis.

erythraea. Its type 1 polyketide synthase (PKS) is able to carry out 20 steps required to form the complex carbon backbone of 6deoxythronolide (1) the advanced precursor of erythromycin A (2) (Schemes 1 and 21). This type 1 polyketide synthases represents an examples of molecular compartmentation. This megaenzyme complex is based on three large proteins that are composed of several enzymes and which can span several so called PKS modules. Altogether, the erythromycin PKS is organised in seven modules (one starting and six extender modules) (Scheme 18).

In each module acyl transferases (AT) choose a starting building block (propionyl-CoA) or six extender units (methylmalonyl-CoA), respectively, and transfer them to the individual acyl carrier proteins (ACP) that play a central role for the following transformations. The ACP-bound intermediates are handed over from one catalytic site to the next and from one module to the next. A set of enzymatic domains (e.g. KS, KR, DH,

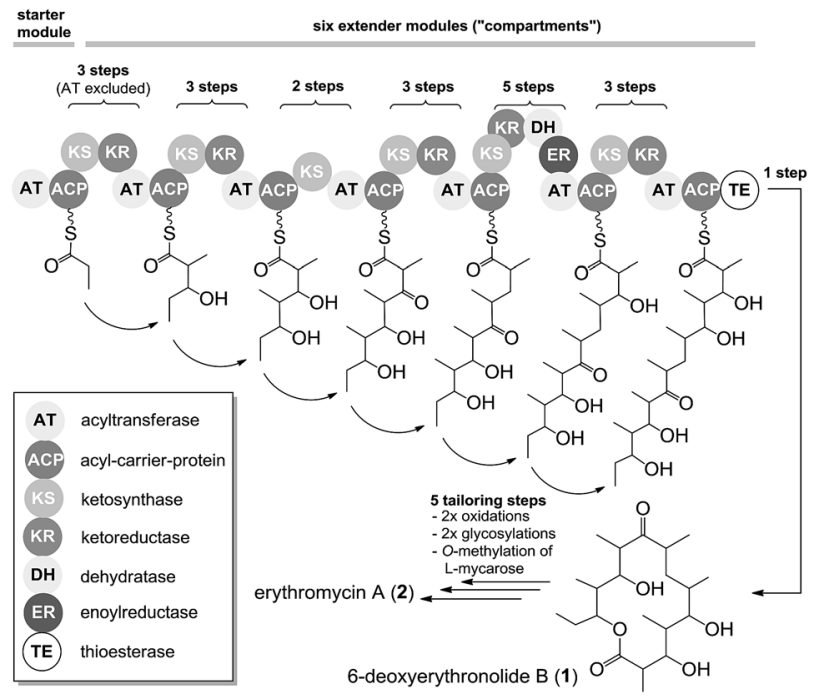

Scheme 18 Biosynthesis of erythromycin A (2). 


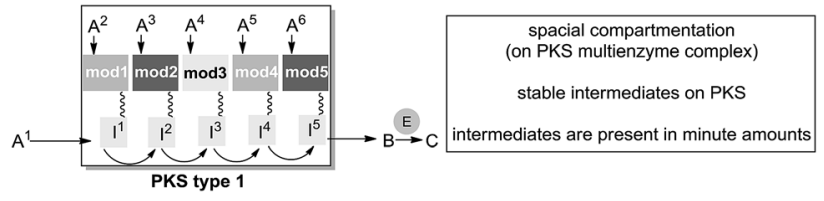

Fig. 7 Polyketide biosynthesis - an example of spatial compartmentation on a molecular level.

ER), each module is responsible for C2-homologation and functional group manipulations (keto $\rightarrow$ alcohol $\rightarrow$ alkene $\rightarrow$ alkane) (Fig. 7).

This modular character and the permanent attachment of intermediates to the PKS lead to spatial separation of each chemical transformation. As the PKS operates continuously under steady state conditions all intermediates are only present in minute amounts (in theory one per protein).

Besides the ribosomal machinery, the PKS I assembly line is probably the most sophisticated megaenzyme architecture nature has developed. It is highly modular and in order to smoothly operate the interaction of neighbouring catalytic domains are perfectly orchestrated. Determining the dynamic architecture of the PKS type I is still difficult due to the large size and high complexity. Staunton and Leadlay developed a 'double-helical' model, that is based on the dimeric structure of subunits (Fig. 8). ${ }^{49}$ The model suggests that two identical proteins are wrapped against each other, which results in a lineup allowing all subunits to interact with one specific ACP domain. The underlying chemical concept of the PKS is compartmentation of single enzymatic steps and close spatial proximity that allow transfer of intermediates from one enzymatic domain to the next in a highly controlled manner (Fig. 8). The twisting peptide chain model also explains why the ACP and KS domains, which require a close proximity in order to allow condensation, are usually found at opposite ends of the peptide chain (Fig. 8, left-hand side). It also explains how a

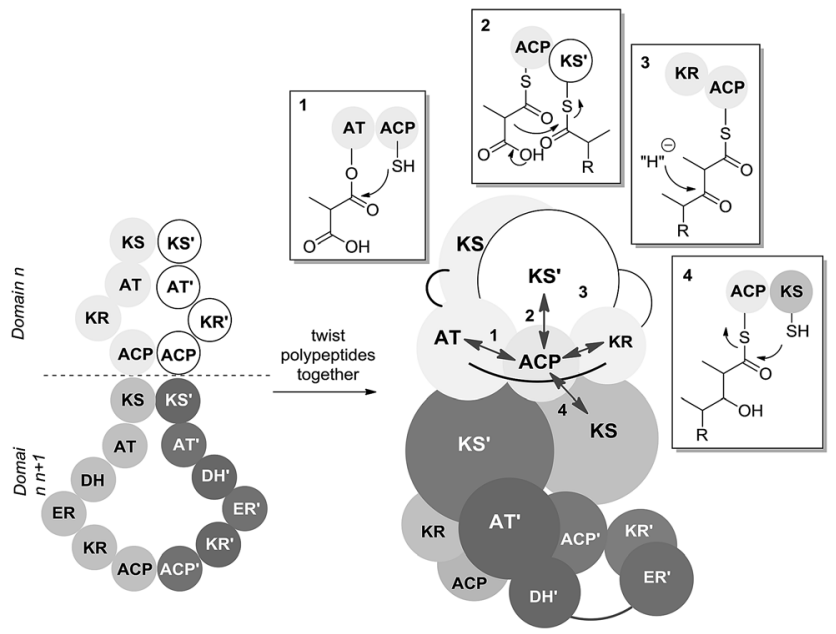

Fig. 8 'Double-helical' model for modular polyketide synthases (PKs) The ACP is situated in the center to allow interaction with neighbouring domains AT, KS', KR and KS. seemingly single module can be used more than once, since a dimer could be used twice in theory, if the folding is favorable. Conceptually, the PKS multistep process resembles a domino reaction with the difference that stable and not high energy intermediates are transformed from one module to the next.

While the chemical approaches towards erythromycin A (2) relied on multistep batch processes (see Scheme 1) the biosynthesis of erythromycin A (2) is a continuous steady state process in which stable intermediates are passed from one spatial environment to the next and in each compartment (module) a new composition of enzymatic activities provides a choice for individual elongation chemistry. Clearly, this free choice does not exist for domino-type multistep transformations as discussed for terpene cyclases and their reactive intermediates.

Conceptually, there is a counterpart in synthetic chemistry. Multistep flow synthesis using linearly assembled flow devices resembles such a continuous compartmented process. ${ }^{50}$ Indeed, continuous flow processes are the best technical option to achieve spatial separation of individual transformations and the individual reactor may be regarded as an individual PKS module (Scheme 21 and Fig. 9). In continuously operated multistep flow synthesis the burden of work-up is minimised and isolation protocols ideally take place after the last step. Furthermore, it is possible to set up flow systems in which two short linear synthetic flow sequences converge for allowing production of structurally complex products from two fragments. Technically, this concept is well established. E.g. one technical way to produce acrylic acid proceeds as a truly multistep continuous process. It starts with the steam cracking process of crude oil followed by a two step catalytic oxidation of propene via acrolein to acrylic acid. It is estimated that 5 to $10^{6}$ tons of acrylic acid are produced worldwide much of it in continuously. ${ }^{51}$

Commonly, intermediates formed in continuous flow processes are chemically stable enough so that other components can be added to the stream of the reaction mixture and conditions required for the next reaction can be adapted in the next reactor (Fig. 9). These options make multistep flow processes more flexible compared to multicomponent or cascade reactions.

A state-of-the-art multistep flow protocol was reported by the McQuade group in 2009. Their three-step flow synthesis of

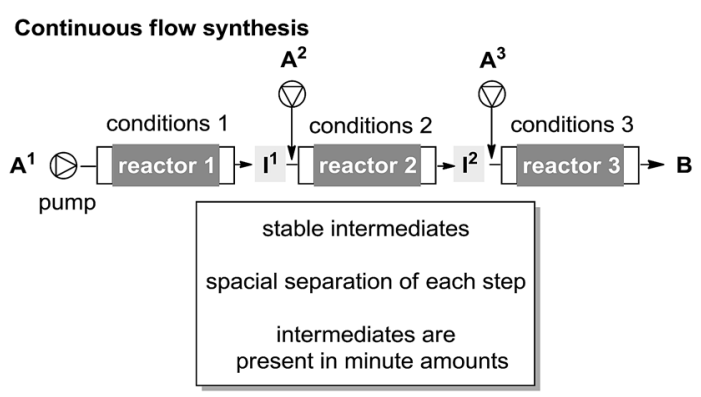

Fig. 9 Principal concept of a multistep flow synthesis of product $B$ starting from educt $A^{1}\left(I^{1}, I^{2}=\right.$ intermediates, $A^{2}, A^{3}=$ additional reactants) 


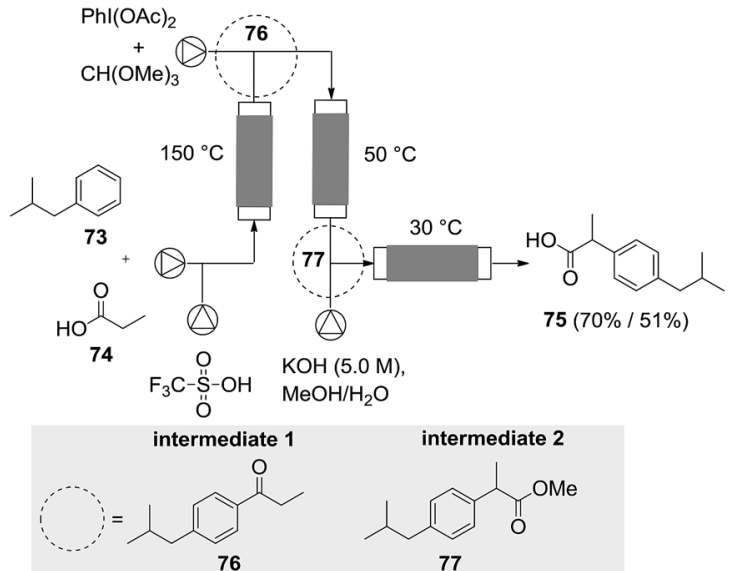

Scheme 19 McQuade's continuous three-step flow process of ibuprofen (75).

ibuprofen (75), an anti-inflammatory and pain relieving drug, was performed continuously without purifying intermediates 76 or 77 (Scheme 19). ${ }^{52}$ The combined streams of neat isobutyl benzene (73), propionic acid (74) and triflic acid provided intermediate 76 (residence time: $5 \mathrm{~min}$ ). Next, 76 was mixed with trimethyl orthoformate (TMOF) and iodosobenzene diacetate by a third stream which initiated the desired 1,2 aryl migration and formation of intermediate 77 . Finally, saponification provided Ibuprofen (75) which had to be purified by column chromatography $(70 \%)$ or by simple recrystallisation of the crude solid (51\%).

A second illustrative example, the continuous multistep flow synthesis of olanzapine (Zyprexa, 82), was recently reported by our own laboratories (Scheme 20). ${ }^{53}$ A continuous three-step synthesis of thieno[1,5]-benzodiazepine $\mathbf{8 0}$ started from aryl iodide 78 and thiophene $\mathbf{7 9}$ which were coupled via a Buchwald-Hartwig amination. The resulting intermediate 83 first had to be passed through an in-line extractor for removing base-

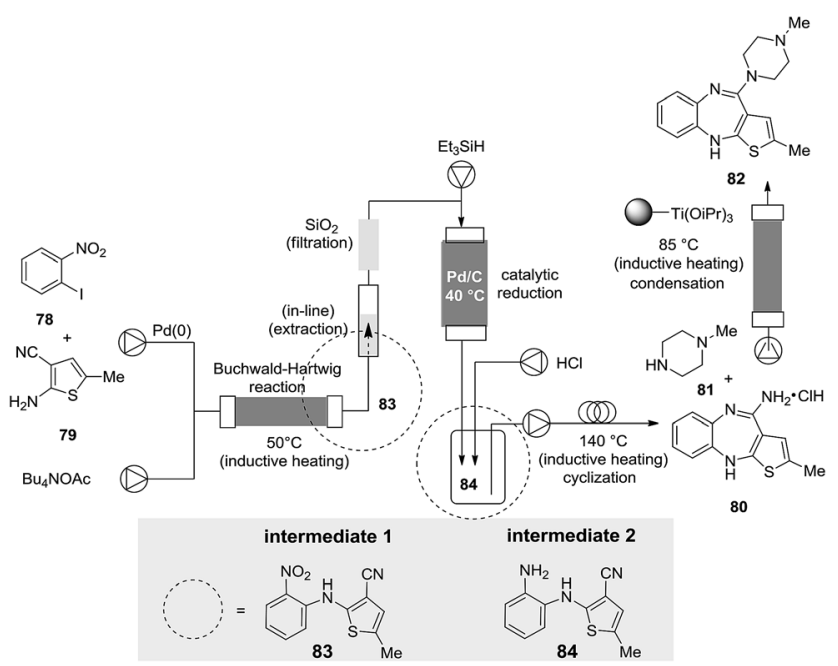

Scheme 20 The continuous multistep flow process of olanzapine (Zyprexa) (82).

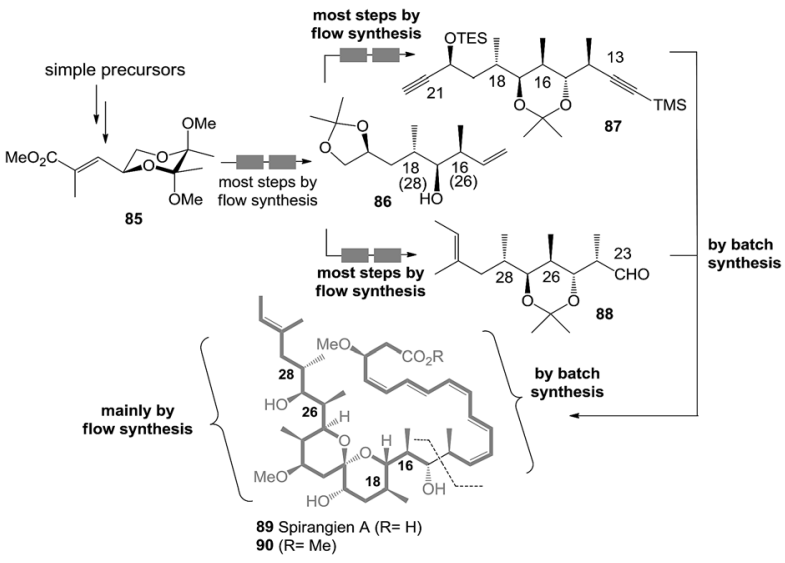

Scheme 21 Ley's total synthesis of the methyl ester of spirangien A (90). ${ }^{53}$

derived ammonium salts then through a pad of silica that trapped palladium black before hydrogenation of the nitro group occurred in the second reactor. The aniline intermediate 84 that left this reactor was collected in a glass vessel where remaining hydrogen gas was liberated. At this point a stream of $\mathrm{HCl}$ in $\mathrm{MeOH}$ was added and the solution was injected into the tubular reactor that was heated inductively. As a result thieno $[1,5]$-benzodiazepine 80 was collected after 30 hours of continuous operation in $88 \%$ yield without the need of chromatographic purification. Finally, the synthesis of olanzapine $\mathbf{8 2}$ was achieved in $83 \%$ yield after coupling with piperazine 81 using an inductively heated fixed bed reactor containing a solid-phase bound titanium Lewis acid.

A most remarkable work was published earlier this year by Ley and coworkers. ${ }^{54}$ During the total synthesis of the methyl ester of spirangien A (90, Scheme 21$)$ a polyketide-based natural product isolated from myxobacteria Sorangium cellulosum So ce90 several polyketide fragments and hence the western part were synthesised under continuous multistep flow conditions without isolation of intermediates.

Strategically, intermediate $\mathbf{8 5}$ was transformed into the key building block 86. It contains a stereotriade that is found at C16-C18 and C26-C28, respectively, of spirangien A (89). Consequently, alkene 86 was transformed into the two advanced fragments $\mathbf{8 7}$ and $\mathbf{8 8}$. These were merged convergently by nucleophilic addition of the alkynyl lithium derivative derived from $\mathbf{8 7}$ to the aldehyde present in $\mathbf{8 8}$. At this point the authors switched from an overall flow process to the batch mode. The synthesis contains a lot of technical details and synthetic subtleties, so that only some key features of individual reactions and reaction sequences can be highlighted which demonstrate the state of the art of modern flow synthesis (Scheme 22).

Utilizing the technically simple device of a tube-in-tube reactor suitable for continuous reactions with gases, the Ley group conducted an asymmetric hydrogenation using Pfaltz's catalyst $(R, R)-\mathbf{9 1}$ with alkene $\mathbf{8 5}$, which yielded hydrogenation product 92 in excellent yield and with good selectivity (Scheme 22). The catalyst's special feature is associated with the fact that 


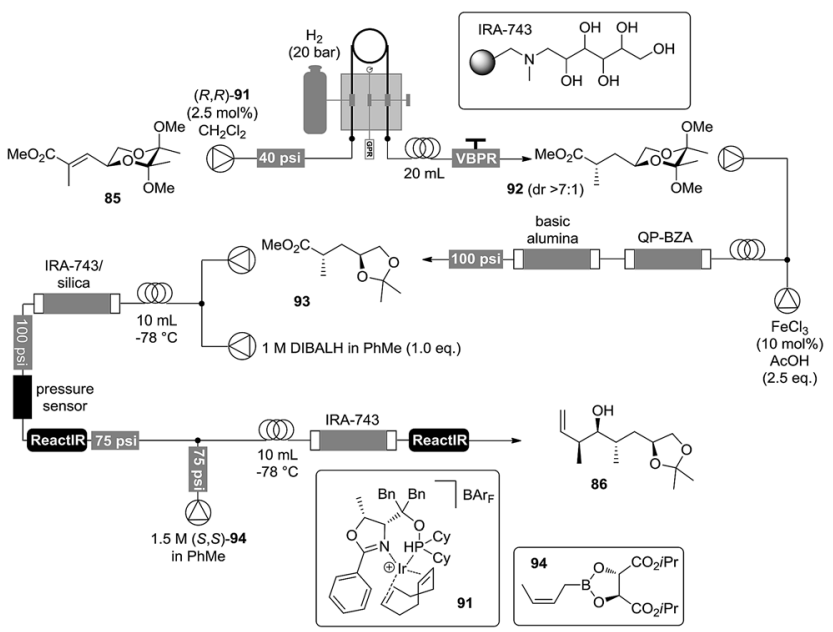

Scheme 22 Multistep flow syntheses of polyketide fragment $86 .^{53}$ $\mathrm{VBPR}=$ variable back pressure regulator; $\mathrm{QP}=$ QuadraPure; $\mathrm{BZA}=$ benzyl amine $\mathrm{BAr}_{\mathrm{F}}=$ tetrakis[3,5-bis(trifluoromethyl)phenyl]borate.

it can be applied for the hydrogenation of non functionalised alkenes without the need of additional polar coordinating sites. ${ }^{55}$ In the following, the protecting group was switched to an acetonide and all reagents and byproducts were removed from product 92 by pumping the reaction mixture through a cartridge of polymer supported benzylamine and basic alumina oxide. Finally, the resulting ester $\mathbf{9 3}$ was selectively reduced to yield the corresponding aldehyde which was followed by the experimentally challenging Roush crotylation with $Z$-but-2-enylboronate $\mathbf{9 4}$ to furnish fragment $\mathbf{8 6}$. It is noteworthy that aluminum containing byproducts were removed from the stream using a fixed bed material composed of a polyalcohol immobilised on the polymer IRA-743. It is now possible to perform the Roush crotylation directly by addition of the boronate to the flow system. Control of stoichiometry was achieved by measuring the concentration of the resulting aldehyde using a FlowIR cell. Data processing, allowed automatic adjustment of the flow rate of the pump that is responsible for the addition of boronate $\mathbf{9 4}$.

It has to be stressed, that in several aspects this total synthesis is not biomimetic, although continuous processes and compartmentation were exploited. Ley's synthesis does not follow the linear logic of the polyketide synthase but has the flexibility to dissect the spirangien A backbone into three major fragments which are merged in a convergent fashion, instead. ${ }^{56}$

\section{Outlook}

This conceptual review has examined the ideal multistep synthesis by analyzing nature. This article is not about biomimetic natural product synthesis but we rather suggest that the key to efficiency is steady state processing with intermediates that are immediately transformed to the next intermediate when formed until the target secondary metabolite is formed. We discussed four strategies (multicatalysis, domino reactions, iteration and compartmentation) that commonly proceed via short-lived intermediates. Nature, unlike chemists, uses these

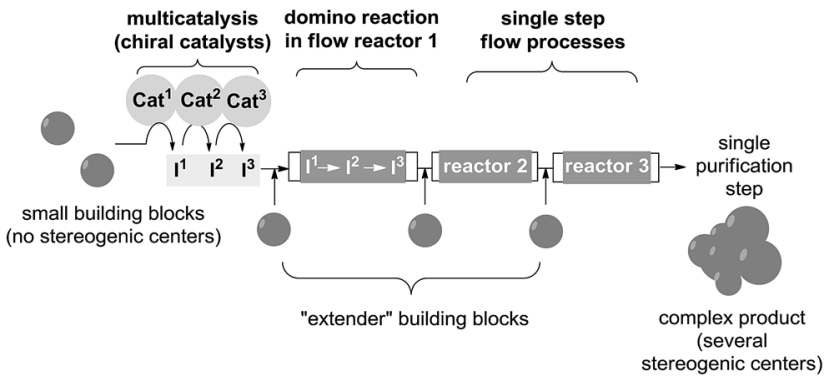

Scheme 23 Multistep processing towards complex molecular targets by combination of different steady state techniques.

four strategies flexibly, almost combinatorially like a juggler which is still a huge challenge for synthetic chemists. In essence, we believe that atom, step and redox-economy can only cover some major aspects of defining the ideal synthesis.

The flexible use of these and additional strategies ${ }^{21}$ should increase the efficiency of processes by minimizing work-up protocols of intermediates so that isolation of the final product is the only purification issue.

We believe that harnessing and enhancing these strategies should not only be a priority for academia but also for industry. We envision that a combination of these methods may one day change 'traditional' synthetic chemistry and open up rapid access to a world of natural product-like compounds with astonishing complexity with only one or two purification steps (Scheme 23). We are aware that it is still a long way to go to develop such processes, but we believe we should not shy from such an opportunity to access a larger part of the chemical space in a more economical fashion.

Synthetic chemists can learn from nature by closely studying - not only individual transformations - but also how in vivo multistep processes are conducted. With the multitude of reactions discovered since Wöhler's first preparation of urea in 1828, synthetic chemists have the potential to develop more powerful multistep processes than nature, if only we can manage to put them into use effectively.

\section{Notes and references}

1 J. B. Hendrickson, J. Am. Chem. Soc., 1975, 97, 5784-5800.

2 B. M. Trost, Science, 1991, 254, 1471-1477.

3 (a) P. A. Wender, M. P. Croatt and B. Witulski, Tetrahedron, 2006, 62, 7505-7511; (b) P. A. Wender, V. A. Verma, T. J. Paxton and T. H. Pillow, Acc. Chem. Res., 2008, 41, 4049; (c) P. A. Wender and B. L. Miller, Nature, 2009, 460, 197-201; (d) P. A. Wender, Nat. Prod. Rep., 2014, 31, 433-440.

4 (a) J. M. Richter, Y. Ishihara, T. Masuda, B. W. Whitefield, T. Llamas, A. Pohjakallio and P. S. Baran, J. Am. Chem. Soc., 2008, 130, 17938-17945; (b) N. Z. Burns, P. S. Baran and R. W. Hoffmann, Angew. Chem., Int. Ed., 2009, 48, 2854-2867.

5 T. Gaich and P. Baran, J. Org. Chem., 2010, 75, 4657-4673. 6 (a) Biomimetic Organic Synthesis, ed. B. Nay and E. Poupon, Wiley-VCH, Weinheim, 2011, vol. 1/2; (b) D. Enders and 
A. A. Narine, J. Org. Chem., 2008, 73, 7857-7870; (c) P. G. Bulger, S. K. Bagal and R. Marquez, Nat. Prod. Rep., 2008, 25, 254-297; (d) A. A. Roberts, K. S. Ryan, B. S. Moore and T. A. M. Gulder, Top. Curr. Chem., 2010, 297, 149-220; (e) M. Razzak and J. K. de Brabander, Nat. Chem. Biol., 2011, 7, 865-875.

7 We do not discuss the important class of alkaloids, because they are not biosynthesised through a common biosynthetic pathway.

8 (a) J. Staunton, P. Caffrey, J. F. Aparicio, G. A. Roberts, S. S. Bethell and P. F. Leadlay, Nat. Struct. Biol., 1996, 3, 188-196; (b) K. J. Weissman and P. F. Leadley, Nat. Rev. Microbiol., 2005, 3, 925-936.

9 (a) R. B. Woodward, E. Logusch, K. P. Nambiar, K. Sakan, D. E. Ward, B.-W. Au-Yeung, P. Balaram, L. J. Browne, P. J. Card, C. H. Chen, R. B. Chênevert, A. Fliri, K. Frobel, H.-J. Gais, D. G. Garratt, K. Hayakawa, W. Heggie, D. P. Hesson, D. Hoppe, I. Hoppe, J. A. Hyatt, D. Ikeda, P. A. Jacobi, K. S. Kim, Y. Kobuke, K. Kojima, K. Krowicki, V. J. Lee, T. Leutert, S. Malchenko, J. Martens, R. S. Matthews, B. S. Ong, J. B. Press, T. V. Rajan Babu, G. Rosseau, H. M. Sauter, M. Suzuki, K. Tatsuta, L. M. Tolbert, E. A. Truesdale, I. Uchida, Y. Ueda, T. Uyehara, A. T. Vasella, W. C. Vladuchick, P. A. Wade, R. M. Williams and H. N.-C. Wong, J. Am. Chem. Soc., 1981, 103, 3210-3213; (b) R. B. Woodward, E. Logusch, K. P. Nambiar, K. Sakan, D. E. Ward, B.-W. Au-Yeung, P. Balaram, L. J. Browne, P. J. Card, C. H. Chen, R. B. Chênevert, A. Fliri, K. Frobel, H.-J. Gais, D. G. Garratt, K. Hayakawa, W. Heggie, D. P. Hesson, D. Hoppe, I. Hoppe, J. A. Hyatt, D. Ikeda, P. A. Jacobi, K. S. Kim, Y. Kobuke, K. Kojima, K. Krowicki, V. J. Lee, T. Leutert, S. Malchenko, J. Martens, R. S. Matthews, B. S. Ong, J. B. Press, T. V. Rajan Babu, G. Rosseau, H. M. Sauter, M. Suzuki, K. Tatsuta, L. M. Tolbert, E. A. Truesdale, I. Uchida, Y. Ueda, T. Uyehara, A. T. Vasella, W. C. Vladuchick, P. A. Wade, R. M. Williams and H. N.-C. Wong, J. Am. Chem. Soc., 1981, 103, 3213-3215; (c) R. B. Woodward, E. Logusch, K. P. Nambiar, K. Sakan, D. E. Ward, B.-W. Au-Yeung, P. Balaram, L. J. Browne, P. J. Card, C. H. Chen, R. B. Chênevert, A. Fliri, K. Frobel, H.-J. Gais, D. G. Garratt, K. Hayakawa, W. Heggie, D. P. Hesson, D. Hoppe, I. Hoppe, J. A. Hyatt, D. Ikeda, P. A. Jacobi, K. S. Kim, Y. Kobuke, K. Kojima, K. Krowicki, V. J. Lee, T. Leutert, S. Malchenko, J. Martens, R. S. Matthews, B. S. Ong, J. B. Press, T. V. Rajan Babu, G. Rosseau, H. M. Sauter, M. Suzuki, K. Tatsuta, L. M. Tolbert, E. A. Truesdale, I. Uchida, Y. Ueda, T. Uyehara, A. T. Vasella, W. C. Vladuchick, P. A. Wade, R. M. Williams and H. N.-C. Wong, J. Am. Chem. Soc., 1981, 103, 3215-3217.

10 X. Gao, S. L. Woo and M. J. Krische, J. Am. Chem. Soc., 2013, 135, 4223-4226.

11 H. C. Lynch and M. E. Bushell, Microbiology, 1995, 141, 31053111.

12 The fermentation yield is based on the average dilution of $0.07 \mathrm{~h}^{-1}$ in a cyclic fed batch with the specific production rate of $0.75 \mathrm{mg} \mathrm{g}^{-1} \mathrm{~h}^{-1}$ by addition of $6 \mathrm{~g} \mathrm{~L}^{-1}$ glucose medium and a constant dilution of $2 \mathrm{~g} \mathrm{~L}^{-1}$ biomass on the one hand and on a chemostat cultivation with a dilution rate of $0.07 \mathrm{~h}^{-1}$ using otherwise the same parameters and a specific production rate of $0.31 \mathrm{mg} \mathrm{g}^{-1} \mathrm{~h}^{-1}$ as reported in ref. 11. It must be noted that exact fermentation conditions and yields of the continuously operated industrial process are not available.

13 (a) A. K. El-Sayed, J. Hothersall, S. M. Cooper, E. Stephens, T. J. Simpson and C. M. Thomas, Chem. Biol., 2003, 10, 419-430; (b) C. M. Thomas, J. Hothersall, C. L. Willi and T. J. Simpson, Nat. Rev. Microbiol., 2010, 8, 281-289.

14 Convergency is not unknown in biosynthesis as exemplified for the biosynthesis of erythromycin A (2). The rare deoxysugars L-mycarose ( $O$-methyation to yield L-cladinose is a tailouring step) and D-desosamine are attached to the aglycon at a late stage and both are biosynthesised in seven steps from D-glucose.

15 J. K. Chakrabarti and D. E. Tupper, Patent DE 2552403 C2, published: 19 June 1986.

16 N. Bhana, R. H. Foster, R. Olney and G. L. Plosker, Drugs, 2001, 61, 111-161.

17 (a) J. Gerlach and L. Peacock, Int. Clin. Psychopharmacol., 1995, 10, 39-48; (b) G. P. Reynolds, J. Psychopharmacol., 2004, 18, 340-345.

18 J. M. McGuire, R. L. Bunch, R. C. Anderson, H. E. Boaz, E. H. Flynn, M. Powell and J. W. Smith, Antibiot. Chemother., 1952, 2, 281-283.

19 In the past two decades molecular biology and genetic engineering has contributed tremendously in deciphering biosynthetic pathways.

20 Review on terpene cyclase: Y. Gao, R. B. Honzatko and R. J. Peters, Nat. Prod. Rep., 2012, 29, 1153-1175.

21 (a) T. Kutchan and R. A. Dixon, Curr. Opin. Plant Biol., 2005, 8, 227-229; (b) K. Yazaki, A. Sugiyama, M. Morita and N. Shitan, Phytochem. Rev., 2008, 7, 513-524.

22 T. J. Burkey and D. Griller, J. Am. Chem. Soc., 1985, 107, 246249.

23 For simplification, other topics such as diversification from a common advanced intermediate (see T. Newhouse, P. S. Baran and R. W. Hoffmann, Chem. Soc. Rev., 2009, 38, 3010-3021 and R. D. Firn and C G. Jones, Nat. Prod. Rep., 2003, 20, 382-391) or multicomponent reactions (MCR) are skipped. In fact, the latter is hardly found in nature.

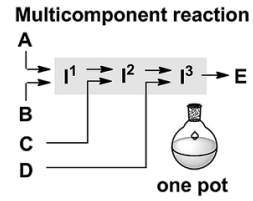

24 (a) W. Eisenreich, M. Schwarz, A. Cartayrade, D. Arigoni, M. H. Zenk and A. Bacher, Chem. Biol., 1998, 5, R221R233; (b) P. M. Dewick, Nat. Prod. Rep., 2002, 19, 181-222.

25 For general reviews see: $(a)$ A. Bruggink, R. Schoevaart and T. Kieboom, Org. Process Res. Dev., 2003, 7, 622-640; (b) J. M. Lee, Y. Na, H. Han and S. Chang, Chem. Soc. Rev., 
2004, 33, 302-312; (c) J. C. Wasilke, S. J. Obrey, R. T. Baker and G. C. Bazan, Chem. Rev., 2005, 105, 1001-1020; (d) L. M. Ambrosini and T. H. Lambert, ChemCatChem, 2010, 2, 1373-1380; (e) D. B. Ramachary and S. Jain, Org. Biomol. Chem., 2011, 9, 1277-1300; $(f)$ M. E. Abbasov and D. Romo, Nat. Prod. Rep., 2014, 31, 1318-1327.

26 N. Jeong, S. D. Seo and J. Y. Shin, J. Am. Chem. Soc., 2000, 122, 10220.

27 K. L. Jensen, P. T. Franke, C. Arrónzi, S. Kobbelgaard and K. A. Jørgensen, Chem.-Eur. J., 2010, 16, 1750-1753.

28 R. Schoevaart and T. Keiboom, Tetrahedron Lett., 2002, 43, 3399-3400.

29 (a) L. F. Tietze, Chem. Rev., 1996, 96, 115-136; (b) K. C. Nicolaou, D. J. Edmonds and P. G. Bulger, Angew. Chem., Int. Ed., 2004, 43, 160-181; (c) F. Löbermann, P. Mayer and D. Trauner, Angew. Chem., 2010, 122, 63356338.

30 B. T. Ueberbacher, M. Hall and K. Faber, Nat. Prod. Rep., 2012, 29, 337-350.

31 D. J. Tantillo, Nat. Prod. Rep., 2011, 28, 1035-1053.

32 P. B. Schiff, J. Fant and S. B. Horwitz, Nature, 1979, 277, 665667.

33 H. G. Floss and U. Mocek, Biosynthesis of taxol., in TaxolScience and Applications, ed. M. Suffness, CRC Press, Boca Raton, FL, USA, 1995, pp. 191-208.

34 T. D. H. Bugg, in Introduction to enzyme and coenzyme chemistry, John Wiley \& Sons, 2012.

35 R. Boffey, W. Whittingham and J. Kilburn, J. Chem. Soc., Perkin Trans. 1, 2001, 487-496.

36 L. F. Tietze, T. Hungerland, A. Düfert, I. Objartel and D. Stalke, Chem.-Eur. J., 2012, 18, 3286-3291.

37 (a) J. M. Crawford and C. A. Townsend, Nat. Rev. Microbiol., 2010, 8, 879-889; (b) R. J. Cox, Org. Biomol. Chem., 2007, 5, 2010-2026.

38 (a) S. M. Ma, J. W.-H. Li, J. W. Choi, H. Zhou, K. K. M. Lee, V. A. Moorthie, X. Xie, J. T. Kealey, N. A. Da Silva, J. C. Vederas and Y. Tang, Science, 2009, 326, 589-592; (b) W. Xu, Y.-H. Chooi, J. W. Choi, S. Li, J. C. Vederas, N. A. Da Silva and Y. Tang, Angew. Chem., Int. Ed., 2013, 52, 6472-6475.

39 For general function of PKS type II: $(a)$ H. Zhou, Y. Li and Y. Tang, Nat. Prod. Rep., 2010, 27, 839-868.

40 For exemplary cyclisation of decaketides: (a) G. Meurer, M. Gerlitz, E. Wendt-Pienkowski, L. C. Vining, J. Rohr and C. H. Hutchinson, Chem. Biol., 1997, 4, 433-443; (b) S.-E. Wohlert, E. Wendt-Pienkowski, W. Bao and C. R. Hutchinson, J. Nat. Prod., 2001, 64, 1077-1080.

41 (a) B. J. Albert and H. Yamamoto, Angew. Chem., Int. Ed., 2010, 49, 2247-2249; (b) P. B. Brady, B. J. Albert, M. Akakura and H. Yamamoto, Chem. Sci., 2013, 4, 32233231.

42 This is a borderline case, because it could also fit the category of domino reactions.

43 B. J. Albert, Y. Yamaoka and H. Yamamoto, Angew. Chem., Int. Ed., 2011, 50, 2610-2612.
44 (a) J. S. Mynderse and R. E. Moore, Phytochemistry, 1979, 18, 1181-1183; (b) S. Carmeli, R. E. Moore, G. M. Patterson, Y. Mori and M. Suzuki, J. Org. Chem., 1990, 55, 4431-4438; (c) Y. Mori, Y. Kohchi, M. Suzuki, S. Carmeli, R. E. Moore and G. M. Patterson, J. Org. Chem., 1991, 56, 631-637.

45 (a) S. J. Lee, T. M. Anderson and M. D. Burke, Angew. Chem., Int. Ed., 2010, 49, 8860-8886; (b) S. J. Lee, K. C. Gray, J. S. Paek and M. D. Burke, J. Am. Chem. Soc., 2008, 130, 466-468; (c) S. Fujii, S. Y. Chang and M. D. Burke, Angew. Chem., Int. Ed., 2011, 50, 7862-7864; (d) E. M. Woerly, A. H. Cherney, E. K. Davis and M. D. Burke, J. Am. Chem. Soc., 2010, 132, 6941-6943.

46 E. M. Woerly, J. Roy and M. D. Burke, Nat. Chem., 2014, 6, 484-491.

47 In cell biology the term compartmentation comprises organelles in such as mitochondria, lysosomes, the endoplasmic reticulum, the cell nucleus etc. Here we use this term to refer to distinct catalytic domains on megaenzyme complexes such as the PKS. There are true uses of such organelles in biosynthesis - (a) morphine (b) p450 operate in peroxisomes etc.

48 R. van der Heijden, D. I. Jacobs, W. Snoeijer, D. Hallard and R. Verpoorte, Curr. Med. Chem., 2004, 11, 1241-1253.

49 (a) J. Staunton, P. Caffrey, J. F. Aparicio, G. A. Roberts, S. S. Bethell and P. F. Leadlay, Nat. Struct. Biol., 1996, 3, 188-196; (b) K. J. Weissman and P. F. Leadley, Nat. Rev. Microbiol., 2005, 3, 925-936.

50 J. Wegner, S. Ceylan and A. Kirschning, Chem. Commun., 2011, 47, 4583-4592.

$51 \mathrm{H}$. J. Arpe, Industrial Organic Chemistry, Wiley-VCH, Weinheim, 6th edn, 2007.

52 (a) R. Bogdan, S. L. Poe, D. C. Kubis, S. J. Broadwater and D. T. McQuade, Angew. Chem., Int. Ed., 2009, 48, 85478550; $(b)$ During the evaluation of this manuscript the Jamison group reported on a second continuous synthesis of ibuprofen: D. R. Snead and T. F. Jamison, Angew. Chem., Int. Ed., 2015, 54, 983-987.

53 J. Hartwig, S. Ceylan, L. Kupracz, L. Coutable and A. Kirschning, Angew. Chem., 2013, 125, 9995-9999; Angew. Chem., Int. Ed., 2013, 52, 9813-9817.

54 S. Newton, C. F. Carter, C. M. Pearson, L. d. C. Alves, H. Lange, P. Thansandote and S. V. Ley, Angew. Chem., 2014, 126, 5015-5020; Angew. Chem., Int. Ed., 2014, 53, 4915-4920.

55 (a) J. Blankenstein and A. Pfaltz, Angew. Chem., Int. Ed., 2001, 40, 4445-4447; Angew. Chem., 2001, 113, 4577-4579; (b) A. Lightfoot, P. Schnider and A. Pfaltz, Angew. Chem., 1998, 110, 3047-3050; Angew. Chem., Int. Ed., 1998, 37, 2897-2899.

56 Other important contributions of multistep flow synthesis in the preparation of drugs are: (a) L. Kupracz and A. Kirschning, Adv. Synth. Catal., 2013, 35, 3369-3374, amitriptyline; (b) P. Zhang, M. G. Russell and T. F. Jamison, Org. Process Res. Dev., 2014, 18, 1567-1570, rufinamide. 